\title{
Search for third-generation leptoquarks and scalar bottom quarks in pp collisions at $\sqrt{s}=7 \mathrm{TeV}$
}

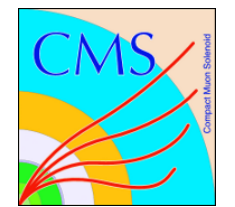

\section{The CMS collaboration}

E-mail: cms-publication-committee-chair@cern.ch

ABSTRACT: Results are presented from a search for third-generation leptoquarks and scalar bottom quarks in a sample of proton-proton collisions at $\sqrt{s}=7 \mathrm{TeV}$ collected by the CMS experiment at the LHC, corresponding to an integrated luminosity of $4.7 \mathrm{fb}^{-1}$. A scenario where the new particles are pair produced and each decays to a b quark plus a tau neutrino or neutralino is considered. The number of observed events is found to be in agreement with the standard model prediction. Upper limits are set at $95 \%$ confidence level on the production cross sections. Leptoquarks with masses below $\sim 450 \mathrm{GeV}$ are excluded. Upper limits in the mass plane of the scalar quark and neutralino are set such that scalar bottom quark masses up to $410 \mathrm{GeV}$ are excluded for neutralino masses of $50 \mathrm{GeV}$.

KEYwORDS: Hadron-Hadron Scattering

ArXiv EPrint: 1210.5627 


\section{Contents}

1 Introduction 1

2 The CMS apparatus 3

3 Razor variables 3

4 Data samples, triggers, and event selection 4

4.1 Muon and electron identification and selection 5

4.2 Identification of $\mathrm{b}$ jets 6

$\begin{array}{llr}5 & \text { Search strategy } & 6\end{array}$

6 Background estimation $\quad 8$

6.1 The $\mathrm{W} / \mathrm{Z}+$ jets background $\quad 9$

$\begin{array}{ll}6.2 \mathrm{t} \overline{\mathrm{t}}+\mathrm{jets} \text { background estimation } & 9\end{array}$

$\begin{array}{lll}6.3 \text { Multijet background } & 10\end{array}$

$\begin{array}{lll}6.4 & \text { Systematic uncertainties } & 12\end{array}$

$\begin{array}{ll}\text { 6.5 ELE control region } & 13\end{array}$

$\begin{array}{lll}7 & \text { Results } & 15\end{array}$

$\begin{array}{lll}8 & \text { Summary } & 20\end{array}$

$\begin{array}{ll}\text { The CMS collaboration } & 25\end{array}$

\section{Introduction}

Many theoretical extensions of the standard model (SM) predict the existence of colortriplet scalar or vector bosons, called leptoquarks (LQ), that have fractional electric charge and both lepton and baryon quantum numbers. These theories include grand unified theories [1], composite models [2, 3], technicolor schemes [4-6], and superstring-inspired $E_{6}$ models [7]. We follow the usual assumption that there are three generations of LQs, each of which couples only to the corresponding generation of SM particles, to avoid violating the known experimental constraints on flavor-changing neutral currents [8]. Leptoquarks would be produced at the Large Hadron Collider (LHC) in pairs predominantly through gg fusion and $\mathrm{q} \overline{\mathrm{q}}$ annihilation, and the contributions from lepton $t$-channel exchange are suppressed by the leptoquark Yukawa couplings. A leptoquark decays to a charged lepton and a quark with a branching fraction $\beta$ usually considered as a free parameter of the model, or a neutrino and a quark with branching fraction $1-\beta$. For scalar LQs, the production 
cross section is determined by the ordinary color coupling between an LQ and a gluon, which is model independent.

Numerous theories of particle physics beyond the SM address the gauge hierarchy problem and other shortcomings of the SM by introducing a new symmetry that relates fermions and bosons, called "supersymmetry" (SUSY) [9]. Supersymmetric models introduce a new discrete symmetry, R-parity, and all SM particles have $R_{p}=+1$ while all superpartners have $R_{p}=-1$. Imposing R-parity conservation prohibits baryon and lepton number violating couplings which could otherwise lead to rapid proton decay. In models with R-parity conservation, SUSY particles are produced in pairs, and the lightest SUSY particle (LSP) is stable. In some models the LSP is the electrically neutral and weakly interacting neutralino $\left(\widetilde{\chi}_{1}^{0}\right)$, which provides a dark matter candidate [10]. The left- and right-handed SM quarks have scalar partners $\left(\tilde{\mathrm{q}}_{L}\right.$ and $\tilde{\mathrm{q}}_{R}$ ) that can mix to form scalar quarks (squarks) with mass eigenstates $\tilde{\mathrm{q}}_{1,2}$. Since the mixing is proportional to the corresponding SM fermion masses, the effects can be enhanced for the third generation squarks, yielding sbottom $\left(\tilde{b}_{1,2}\right)$ and stop $\left(\tilde{\mathrm{t}}_{1,2}\right)$ mass eigenstates with large mass splitting. The lighter mass eigenstate $\left(\tilde{\mathrm{b}}_{1}\right.$ or $\tilde{\mathrm{t}}_{1}$ ) could be lighter than any other charged SUSY particle [11]. Therefore, if sufficiently light, $\widetilde{\mathrm{b}}_{1}$ squarks could be produced at the LHC either directly or through decays of gluinos (the supersymmetric partners of gluons). In most SUSY models, a $\widetilde{b}_{1}$ is expected to decay predominantly into a bottom quark and $\widetilde{\chi}_{1}^{0}$, so that the final state consists of b jets and a sizable imbalance in transverse energy $\left(\mathbb{E}_{\mathrm{T}}\right)$, defined as the magnitude of the vector opposite to the sum of the transverse momenta of all detected particles.

In this paper we present results of a search for pair-produced scalar third-generation leptoquarks $\left(\mathrm{LQ}_{3}\right)$ with an electric charge of $\pm 1 / 3$ and for $\tilde{b}_{1}$. Each of the $\mathrm{LQ}_{3}\left(\tilde{\mathrm{b}}_{1}\right)$ particles decays into a b quark and $\nu_{\tau}\left(\widetilde{\chi}_{1}^{0}\right)$. In each case, signal events are characterized by two high-transverse-momentum $\left(p_{\mathrm{T}}\right)$ b jets accompanied by large $\mathbb{E}_{\mathrm{T}}$. The resulting final state, consisting of jets, $\mathbb{E}_{\mathrm{T}}$, and no charged leptons, does not allow a full reconstruction of the decay chain, because of the lack of knowledge of the individual momenta of the weakly interacting particles.

Previous searches performed by the CDF and D0 collaborations at the Tevatron have excluded $\mathrm{LQ}_{3} \rightarrow \nu_{\tau} \bar{b}$ masses below $247 \mathrm{GeV}$, and set limits on the production of $\widetilde{\mathrm{b}}_{1}$ squarks for a range of values in the $\widetilde{\mathrm{b}}_{1}-\widetilde{\chi}_{1}^{0}$ mass plane that extend up to $m\left(\widetilde{\mathrm{b}}_{1}\right)=200 \mathrm{GeV}$ for $m\left(\widetilde{\chi}_{1}^{0}\right)=110 \mathrm{GeV}[12,13]$. A search performed by the CMS collaboration has excluded the existence of a scalar $\mathrm{LQ}_{3}$ with an electric charge of $\pm 2 / 3$ or $\pm 4 / 3$ and with mass below $525 \mathrm{GeV}$, assuming $100 \%$ branching fraction to a b quark and a $\tau$ lepton [14]. A search performed by the ATLAS collaboration excluded the production of $\widetilde{b}_{1}$ with masses up to $390 \mathrm{GeV}$, for $\widetilde{\chi}_{1}^{0}$ masses below $60 \mathrm{GeV}[15]$.

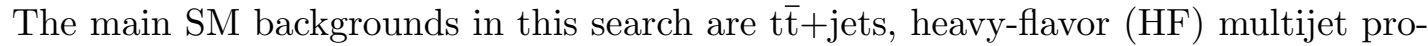
duction, and $\mathrm{W}$ or $\mathrm{Z}$ accompanied by $\mathrm{HF}$ production. In the case of multijet events and $\mathrm{W} / \mathrm{Z}$ decays to hadrons, the $\mathbb{E}_{\mathrm{T}}$ is due to neutrinos in HF semileptonic decays, and due to effects of jet energy resolution and mismeasurements. In the case of $\mathrm{W} / \mathrm{Z}$ decays to leptons, genuine $\mathbb{E}_{\mathrm{T}}$ results from the escaping neutrinos when the charged lepton (e or $\mu$ ) goes undetected, or from $\tau$ decays. 


\section{The CMS apparatus}

A detailed description of the Compact Muon Solenoid (CMS) detector can be found elsewhere [16]. The central feature of the CMS detector is the superconducting solenoid magnet, of $6 \mathrm{~m}$ internal diameter, providing a magnetic field of $3.8 \mathrm{~T}$. The silicon pixel and strip tracker, the lead-tungstate crystal electromagnetic calorimeter (ECAL), and the brass/scintillator hadron calorimeter (HCAL) are contained within the solenoid. Muons are detected in gas-ionization chambers embedded in the steel return yoke. The ECAL has a typical energy resolution of $1-2 \%$ for electrons and photons above $100 \mathrm{GeV}$. The HCAL, combined with the ECAL, measures the jet energy with a resolution $\Delta E / E \approx$ $100 \% / \sqrt{E / \mathrm{GeV}} \oplus 5 \%$.

CMS uses a right-handed coordinate system, with the origin located at the nominal collision point, the $x$ axis pointing towards the center of the LHC ring, the $y$ axis pointing up (perpendicular to the plane of LHC ring), and the $z$ axis along the counterclockwisebeam direction. The azimuthal angle $\phi$ is measured with respect to the $x$ axis in the $x-y$ plane and the polar angle $\theta$ is defined with respect to the $z$ axis. The pseudorapidity is defined as $\eta=-\ln [\tan (\theta / 2)]$.

\section{Razor variables}

Although the signal considered in this analysis consists of two high $p_{\mathrm{T}} \mathrm{b}$ jets and $\mathbb{E}_{\mathrm{T}}$, additional jets may be produced by initial- or final-state radiation (ISR/FSR). We study the effect of such radiation with Monte Carlo (MC) simulation samples. To reduce the systematic uncertainty due to the imperfect simulation of ISR/FSR, we force every event into a dijet topology by combining all the jets in the event into two "pseudojets", following the "razor" methodology and variables $[17,18]$. The pseudojets are constructed as a sum of the four-momenta of their constituent jets. After considering all possible partitions of the jets into two pseudojets, the combination that minimizes the sum in quadrature of the pseudojet masses is selected.

The razor methodology provides an inclusive technique to search for production of heavy particles, each decaying to a visible system of particles and a weakly interacting particle. As an example, let us consider the pair production of two massive particles, denoted $S$, each decaying to a b quark and neutral weakly interacting particle, $\chi$, as $S \rightarrow$ b $\chi$. In the respective rest frame of each particle $S$, the decay products have a unique momentum $p$ resulting from the two-body decay of $S$, given by:

$$
p=\frac{M_{S}^{2}-M_{\chi}^{2}}{2 M_{S}},
$$

where the mass of the $\mathrm{b}$ quark is neglected in this expression. This characteristic momentum, which is denoted $M_{\Delta}$ and is referred to as "momentum scale", is the same in each decay instance, and can be used to distinguish this particular signal from SM backgrounds in the same final states. The razor mass, $M_{R}$, is an event-by-event estimator of this scale calculated through a series of approximations, motivated by physics, meant to estimate the 
rest frames of the respective particles $S[17,18]$, and is defined as:

$$
M_{R} \equiv \sqrt{\left(\left|\overrightarrow{p^{1}}\right|+\left|\overrightarrow{p^{2}}\right|\right)^{2}-\left(p_{z}^{1}+p_{z}^{2}\right)^{2}} \sim 2 M_{\Delta},
$$

where $p^{i}\left(p_{z}^{i}\right)$ is the absolute value (the longitudinal component) of the $i$-th pseudojet momentum. An average transverse mass $M_{\mathrm{T}}^{R}$ can be defined as:

$$
M_{\mathrm{T}}^{R} \equiv \sqrt{\frac{\mathbb{E}_{\mathrm{T}}\left(p_{\mathrm{T}}^{1}+p_{\mathrm{T}}^{2}\right)-{\overrightarrow{E_{\mathrm{T}}}}_{\left(p_{\mathrm{T}}^{1}+\vec{p}_{\mathrm{T}}^{2}\right)}}{2}}
$$

whose maximum value for signal events equals $M_{\Delta}$. The dimensionless variable $R$ is then defined as:

$$
R \equiv \frac{M_{\mathrm{T}}^{R}}{M_{R}}
$$

For the signatures examined in this analysis, the value of $M_{R}$ can have different interpretations. In the case of $\mathrm{LQ}_{3}$ pair production, the $\mathrm{LQ}_{3}$ corresponds to the particle $S$ from the above example, while $\chi$ is a neutrino. As a result, the characteristic scale $M_{\Delta}$ is an estimator of the $\mathrm{LQ}_{3}$ mass. Similarly, for $\widetilde{\mathrm{b}}_{1}$ pair production, $S$ refers to a $\widetilde{\mathrm{b}}_{1}$ while $\chi$ is the LSP, generally a massive neutralino. In this case, $M_{\Delta}$ corresponds to the mass difference between the $\widetilde{b}_{1}$ and LSP.

As follows from the definitions above, $M_{\mathrm{T}}^{R}$ is expected to have a kinematic endpoint at the mass of the new heavy particle, in a similar fashion to the transverse mass having an edge at the particle mass (such as $M_{\mathrm{T}}$ in $\mathrm{W} \rightarrow \ell \nu$ events). Therefore, the $R$ variable is a measure of how well the missing transverse momentum is aligned with respect to the visible momentum. If the missing momentum is completely back-to-back to the visible momentum, $R$ will be close to one. On the other hand, if the momenta of the two neutrinos or $\widetilde{\chi}_{1}^{0}$ largely cancel each other, $R$ will be small. The distribution of $R$ for signal events will peak around 0.5 , while for QCD multijet events it peaks at zero. These properties of $R$ and $M_{R}$ motivate the kinematic requirements for the signal selection and background reduction, which are discussed below.

Some differences between the kinematic distributions (such as the transverse momenta of $\mathrm{b}$ jets) for $\mathrm{LQ}_{3}$ production and $\widetilde{\mathrm{b}}_{1}$ production may arise, if the mass of the $\widetilde{\chi}_{1}^{0}$ is substantial or even almost degenerate with the mass of the $\widetilde{\mathrm{b}}_{1}$. For a fixed $\widetilde{\mathrm{b}}_{1}$ mass the $M_{\Delta}$ decreases as the $\widetilde{\chi}_{1}^{0}$ mass increases. In the case of an almost degenerate $\widetilde{\chi}_{1}^{0}$ and $\widetilde{b}_{1}, \mathbb{E}_{\mathrm{T}}$ is relatively small and the jets are soft, resulting in an $M_{R}$ distribution shifted towards lower values, thus reducing the momentum of the $\widetilde{b}_{1}$ decays products and the sensitivity of the search.

\section{Data samples, triggers, and event selection}

The analysis is designed using MC samples generated with PYTHIA (version 6.424) [19] and MADGRAPH [20] (version 5.1.1.0), and processed with a detailed simulation of the CMS detector response based on GEANT4 [21]. Events with QCD multijets, top quarks, and electroweak bosons are generated with MADGRAPH interfaced with PYTHIA tune Z2 [22] for parton showering, hadronization, and the underlying event description. Signal samples 
for $\mathrm{LQ}_{3}$ masses from 200 to $650 \mathrm{GeV}$, in steps of $50 \mathrm{GeV}$, are generated with PYTHIA tune D6T $[23,24]$. The $\widetilde{b}_{1}$ pair production signal samples are generated with the PYTHIA generator and processed with a detailed fast simulation of the CMS detector response [25]. The scalar bottom quark signal samples are generated with $\widetilde{b}_{1}$ masses from $100 \mathrm{GeV}$ to $550 \mathrm{GeV}$ in steps of $25 \mathrm{GeV}$, and $\widetilde{\chi}_{1}^{0}$ masses from $50 \mathrm{GeV}$ to $500 \mathrm{GeV}$ in steps of $25 \mathrm{GeV}$. The $\widetilde{b}_{1}$ samples are generated with the assumption that the mass peak can be described by a Breit-Wigner shape [19], but this assumption becomes imprecise when the sparticles are close to degenerate. Samples where the difference between the $\widetilde{b}_{1}$ mass and $\widetilde{\chi}_{1}^{0}$ mass is less than $50 \mathrm{GeV}$ are therefore not generated. The simulated events are reweighted so that the distribution of number of overlapping pp interactions per beam crossing ("pileup") in the simulation matches that observed in data.

Events used in this search are collected by a set of online triggers. The first level (L1) of the CMS trigger system, composed of custom hardware processors, uses information from the calorimeters and muon detectors to select the most interesting events in a fixed time interval of less than $4 \mu \mathrm{s}$. The High Level Trigger (HLT) processor farm further decreases the event rate from around $100 \mathrm{kHz}$ to around $300 \mathrm{~Hz}$, before data storage. We employ three categories of triggers for this search: (i) hadronic razor triggers with moderate/tight requirements on $R$ and $M_{R}$; (ii) muon razor triggers with looser requirements on $R$ and $M_{R}$ and at least one muon in the central part of the detector with $p_{\mathrm{T}}>10 \mathrm{GeV}$; and (iii) electron razor triggers with the $R$ and $M_{R}$ requirements similar to those for muon razor triggers, and at least one electron of $p_{\mathrm{T}}>10 \mathrm{GeV}$, satisfying loose isolation criteria. Events collected with the muon and electron razor triggers are used to provide control regions for background studies, since the potential signal contribution in these events is negligible. The search for the presence of a new physics signal is performed in the events collected with the hadronic razor triggers.

All events are required to have at least one good reconstructed interaction vertex [26]. Events containing calorimeter noise, or large $\mathbb{E}_{\mathrm{T}}$ due to instrumental effects (such as beam halo or jets near non-functioning channels in the ECAL) are removed from the analysis [27]. The jets in the event, which are required to have $|\eta|<3.0$, are reconstructed from the calorimeter energy deposits using the infrared-safe anti- $k_{\mathrm{T}}$ algorithm [28] with a distance parameter of 0.5 , and are corrected for the non-uniformity of the calorimeter response in energy and $\eta$ using corrections derived from Monte Carlo and observed data [29]. The $\mathbb{E}_{\mathrm{T}}$ is reconstructed using the particle-flow algorithm, which identifies and reconstructs individually the particles produced in the collision, namely charged hadrons, photons, neutral hadrons, electrons, and muons [30].

\subsection{Muon and electron identification and selection}

We select muon and electron candidates using a cut-based approach similar to the selection process used for the measurement of the inclusive $\mathrm{W}$ and $\mathrm{Z}$ cross section [31].

We use the "tight" and "loose" muon identification criteria, and all muons are required to have $p_{\mathrm{T}}>20 \mathrm{GeV}$. For loose muons, we require that the muon candidate has at least 10 hits in the inner tracker. For the tight muon we require in addition that the following selections are met: 
- at least one hit in the pixel detector;

- impact parameter in the transverse plane $\left|d_{0}\right|<0.2 \mathrm{~cm}$;

- $|\eta|<2.4$.

In addition, the tight muons satisfy a lepton isolation requirement $I_{\text {comb }}$ obtained by summing the $p_{\mathrm{T}}$ of tracks and the energies of calorimetric energy deposits in a cone of $\Delta R=\sqrt{(\Delta \eta)^{2}+(\Delta \phi)^{2}}<0.3$ around the lepton candidate, excluding the candidate's $p_{\mathrm{T}}$. We require the combined isolation to be less than $15 \%$ of the muon $p_{\mathrm{T}}$.

The selection requirements for prompt electrons are:

- $p_{\mathrm{T}}>20 \mathrm{GeV}$ and $|\eta|<2.5$;

- combined isolation $I_{\text {comb }}<15 \%$ of electron $p_{\mathrm{T}}$;

- standard electron identification for barrel (endcap) electrons, defined as follows:

- shape compatible with that of an electron, defined by a measure of the second moment of energy distribution among crystals $\sigma_{\eta \eta}<0.012(0.031)$ [31];

- track-cluster matching in the $\phi$-direction, $\Delta \phi<0.8(0.7)$;

- track-cluster matching in the $\eta$-direction, $\Delta \eta<0.007$ (0.011).

When the isolation requirements [31] are applied to the electron or tight muon candidates, the combined isolation $I_{\text {comb }}$ is corrected for pileup dependence using the average energy density $\rho$ from other proton-proton collisions in the same beam crossing, calculated for each event [32].

\subsection{Identification of $\mathrm{b}$ jets}

Jets originating from a b quark are identified ("tagged") by the TCHE algorithm [33]. Selecting events with b-tagged jets reduces the background from QCD multijet events where mismeasured light-flavor jets cause large apparent $\mathbb{E}_{\mathrm{T}}$. In the TCHE algorithm a jet is considered as $\mathrm{b}$ tagged if there are at least two high-quality tracks within the jet, each with a three-dimensional impact parameter (IP) significance IP $/ \sigma_{\text {IP }}$ larger than a given threshold ("operating point"). In this analysis we use the "medium" operating point [33]. The b-tagging efficiency $\left(\epsilon_{\mathrm{b}}\right)$ and mistag rate $\left(R_{\mathrm{b}}\right)$ have been measured up to $p_{\mathrm{T}}=670 \mathrm{GeV}$ and in the $p_{\mathrm{T}}$ range $80-120 \mathrm{GeV}$ are found to be $\epsilon_{\mathrm{b}}=0.69 \pm 0.01$ and $R_{\mathrm{b}}=0.0286 \pm 0.0003$. In the following we refer to the sample with two jets tagged by the medium TCHE tagger as the "2b-tagged" sample. A scale factor (per jet) of $0.95 \pm 0.02$ is applied to the to the MC simulation samples to account for the observed differences in the b-tagging efficiency between the simulation and data [33].

\section{$5 \quad$ Search strategy}

Candidate signal events in this search contain a pair of b jets, large $\mathbb{E}_{\mathrm{T}}$, and no isolated leptons. The main backgrounds that contribute to this final state originate from $t \bar{t}+j e t s$, 
HF multijets, and $\mathrm{W} / \mathrm{Z}+\mathrm{HF}$ jets events. Diboson production is included in the total background estimation, but its contribution is small. Significant $\mathbb{E}_{\mathrm{T}}$ in multijet events derives from $b$ quarks decaying semileptonically or from jet energies being severely mismeasured. Apart from the multijet background, the remaining backgrounds originate from processes with both genuine $\mathbb{E}_{\mathrm{T}}$ due to energetic neutrinos and undetected charged leptons from vector boson decays.

Data sets collected with the razor triggers are examined for the presence of a wellidentified electron or muon, as described in section 4.1. Based on the presence or absence of such a lepton, the event is categorized into one of the three disjoint event samples (boxes) referred to as the electron (ELE), muon (MU), and hadronic (HAD) boxes.

These requirements define the inclusive baseline selection:

- MU box: events collected with muon razor triggers and containing one loose muon with $p_{\mathrm{T}}>20 \mathrm{GeV}, M_{R}>400 \mathrm{GeV}$ and $R^{2}>0.14$.

- ELE box: events collected with electron razor triggers and containing one loose electron with $p_{\mathrm{T}}>20 \mathrm{GeV}, M_{R}>400 \mathrm{GeV}$ and $R^{2}>0.14$.

- HAD box: events collected with hadronic razor triggers and not satisfying any other box requirements, and with $M_{R}>400 \mathrm{GeV}$ and $R^{2}>0.2$.

We also require that there are at least two jets above $60 \mathrm{GeV}$ in each event, to ensure that the trigger is fully efficient for our selected events. In order to study and estimate the background contributions in the HAD box, we treat muons and electrons in the MU and ELE boxes as neutrinos, i.e. the lepton 4 -vector is used to recalculate the $\mathbb{E}_{\mathrm{T}}$ vector and the $R$ variable is recomputed. This procedure generates the kinematic properties of the background events in the HAD box, using events from the MU and ELE boxes that, because of the presence of the leptons, are free of the signals relevant to this analysis.

The distributions of the discriminating variables $R$ and $M_{R}$ for the main backgrounds (heavy-flavor multijets and $t \bar{t}$ ) are estimated from observed data. Events in the MU box are used to extract the probability density functions (PDFs) describing the behavior of the $R$ and $M_{R}$ shapes for each process of interest. For the $\mathrm{W} / \mathrm{Z}+\mathrm{HF}-$ jets and diboson backgrounds we use heavy-flavor-enriched MADGRAPH simulation samples to get the shape prediction. The procedure to extract the background shapes is described in detail in section 6 , and the samples used are summarized in table 1.

To predict the SM background normalizations in the signal region we adopt the following strategy. The events in the ELE and HAD boxes are split into two exclusive categories:

- sideband: events with $400<M_{R}<600 \mathrm{GeV}$ and $0.2<R^{2}<0.25$;

- high $R^{2}$ : events with $M_{R}>400 \mathrm{GeV}$ and $R^{2}>0.25$.

The $2 \mathrm{~b}$-tagged high- $R^{2}$ events in the HAD box define the signal search region. The normalizations of the SM backgrounds in the signal region are obtained through a two-step procedure: 


\begin{tabular}{|cccc|}
\hline Sample & $R^{2}$ cut & Leptons & Comment \\
\hline W $/ \mathrm{Z} \mathrm{MC}$ & $R^{2}>0.07$ & tight $\mu$ & shape of $\mathrm{W} / \mathrm{Z}+\mathrm{HF}$ jets \\
$\mathrm{MU}$ & $R^{2}>0.14$ & tight $\mu$ & shape of $\mathrm{t} \overline{\mathrm{t}}+$ jets \\
$\mathrm{MU}$ & $R^{2}>0.14$ & loose $\mu$ & shape of HF multijets \\
$\mathrm{ELE}$ & $0.2<R^{2}<0.25$ & tight e & $M_{R}<600$, sideband to extract $f_{R^{2}, \mathrm{ELE}}$ \\
$\mathrm{ELE}$ & $R^{2}>0.25$ & tight e & ELE "signal-like" control region \\
HAD & $0.2<R^{2}<0.25$ & veto leptons & $M_{R}<600$, sideband to extract $f_{R^{2}, \mathrm{HAD}}$ \\
HAD & $R^{2}>0.25$ & veto leptons & signal box, search for signal \\
\hline
\end{tabular}

Table 1. Summary of samples used in the search, with a short description of their specific purpose. Events in all samples are required to have $M_{R}>400 \mathrm{GeV}$ and to include two b-tagged jets. The selections on $R^{2}$ listed in the table are applied after recalculating $\mathbb{E}_{\mathrm{T}}$ and $R$ for events in which charged leptons are treated as neutrinos. The definitions of muons $(\mu)$ and electrons (e) are discussed in section 4.1 .

- the SM processes are normalized according to their theoretical cross sections, except for $\mathrm{t} \overline{\mathrm{t}}$ where the measured CMS cross section [34] is used;

- the total background prediction in the high- $R^{2}$ region is multiplied by a scale factor $\left(f_{R^{2}}\right)$ to correct for imperfect knowledge of the multijet production cross section.

The scale factor is derived from events in the sideband, and is defined as $f_{R^{2}}=$ $N_{\text {exp }} / N_{\text {obs }}$, where $N_{\text {exp }}$ is obtained using the background PDF normalized to their individual cross sections; and $N_{\text {obs }}$ is the number of observed events.

In order to avoid potential bias in the search, before analyzing the events in the HAD box signal region, we test our understanding of the SM background estimation procedure in control regions, using the MU and ELE boxes. This is done by comparing the background shapes derived from the MU box to the observed data in the ELE box (removing the leptons from the reconstruction to emulate $\mathbb{E}_{\mathrm{T}}$ in each case). To ensure that both the shapes and normalizations of the background components describe the observed events, the procedure to be used in the HAD box (see table 1 below) is first employed and tested in the ELE box (section 6.5). Events in the ELE sideband are used to obtain the scale factor $f_{R^{2} \text {, ELE }}$ which is used to test the background prediction in high $R^{2}$ ELE box. Once the procedure

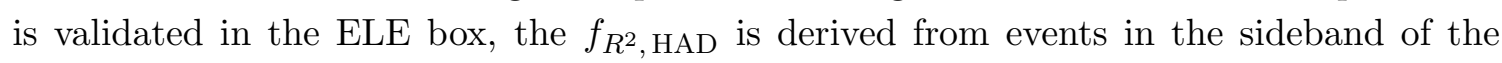
HAD box, and is used to predict the normalization of the backgrounds in the signal region.

\section{Background estimation}

In both simulation and observed data, the distributions of SM background events have been shown to have a simple exponential dependence on the razor variables $R$ and $M_{R}$ over a large fraction of the $R^{2}-M_{R}$ plane $[17,18]$. The shape of the $M_{R}$ tail is well-described by two exponentials with slope parameters $S_{i}(i=1,2)$, where each $S_{i}$ depends linearly on the $R^{2}$ selection threshold $\left(R_{\min }^{2}\right): S_{i}=A_{i}+B_{i} \times R_{\min }^{2}$. 
We construct a simultaneous fit across different $R$ bins, where the $M_{R}$ distribution is fitted for each value of the $R^{2}$ threshold to extract the $A_{i}$ and $B_{i}$ parameters. The simultaneous fit allows one to fully exploit the correlations between the fit parameters and therefore $(i)$ to get a better estimate on the uncertainty of the $A_{i}$ and $B_{i}$ parameters, and (ii) to ensure that the PDF obtained from the fit can be used in regions with various $R^{2}$ thresholds. The functional form used in the fit for a fixed value of the $R$ threshold is:

$$
F\left(M_{R}\right)=\mathrm{e}^{-\left(A_{1}+B_{1} \times R_{\min }^{2}\right) M_{R}}+f \times \mathrm{e}^{-\left(A_{2}+B_{2} \times R_{\min }^{2}\right) M_{R}},
$$

where $f$, the relative amplitude of the second exponent, is extracted from the fit. The values of the shape parameters that maximize the likelihood in the fits, along with the corresponding covariance matrix, are used to define the background model and the uncertainty associated with it. Therefore, if a pure sample of a given process is selected, the PDF describing the behavior of the $R$ and $M_{R}$ shapes of a given process can be extracted.

The fits are performed using the RooFit toolkit [35]. The background PDFs are then used to generate pseudoexperiments, to evaluate the effects of systematic uncertainties on the event yields, as described below in section 6.4.

\subsection{The $\mathrm{W} / \mathrm{Z}+$ jets background}

Owing to the lack of a high-purity data sample enriched in events with $\mathrm{W} / \mathrm{Z}+$ two heavyflavor jets, we estimate the shape of the $\mathrm{W} / \mathrm{Z}+$ jets background using $\mathrm{MC}$ simulated events. A selection of events in the observed data whose jets fail to be b-tagged could provide a sample enriched in $\mathrm{W}+$ light flavor jets. However, because of the b-tagging efficiency on the jet $p_{\mathrm{T}}$ [33], the PDF extracted from these events does not provide a sufficiently accurate model for $\mathrm{W} / \mathrm{Z}+\mathrm{b}$ jets events. Therefore, we estimate the shape of the $\mathrm{W} / \mathrm{Z}+$ jets background using simulated events generated with the MADGRAPH event generator interfaced with PYTHIA, which were found to give an adequate description of CMS observed data $[36,37]$. Residual deficiencies of this MC simulation-based background modeling are accounted for in the extraction of the t $\bar{t}$ background estimate from observed data, as described in the section 6.2. The overall normalization of this background is determined using the observed events in the sideband region of the HAD box.

We perform an unbinned fit of the $\mathrm{W} / \mathrm{Z}+$ jets $M_{R}$ distribution in simulated events passing the MU box selections with $2 \mathrm{~b}$-tagged events, using the sum of two separate exponential terms, as shown in eq. (6.1). The fit allows us to obtain a parametric description of the background that is later used in the derivation of the remaining backgrounds, and it also permits the extrapolation of the prediction into the region of higher $R$ and $M_{R}$ values. The fit is performed in the region $M_{R}>400 \mathrm{GeV}$ and is binned in values of $R^{2}$ as shown in figure 1. The fit to the simulated data, which provides a good description of the $M_{R}$ distribution, is used as the PDF to estimate the $\mathrm{W} / \mathrm{Z}+\mathrm{b}$ jets background in the signal box.

\section{2 $t \bar{t}+$ jets background estimation}

We estimate the $t \bar{t}$ background from the MU box, using 2b-tagged events in collision data (section 4.2) and requiring the presence of a muon passing the tight identification requirements (section 4.1). Based on comparisons with the MC simulation, approximately $90 \%$ 


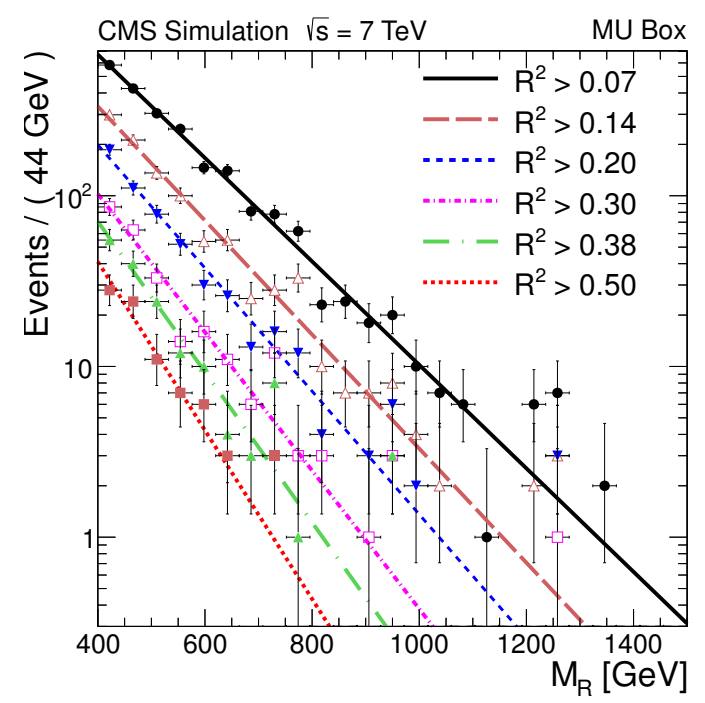

Figure 1. $M_{R}$ distributions for different values of the $R^{2}$ threshold for events passing the MU box selections in the $\mathrm{W} / \mathrm{Z}+$ jets $\mathrm{MC}$ simulation. The results of the fits (lines) are overlaid with the $M_{R}$ distributions from the MC simulation (markers).

of the events in this sample are t $\bar{t}$. We find empirically from MC simulation studies that the shape of the $M_{R}$ distribution in both the tightly selected MU box and in the HAD box is very similar, as can be seen in figure 2. We therefore use the shape derived from the $2 \mathrm{~b}$-tagged sample to predict the $\mathrm{t} \overline{\mathrm{t}}$ background in the signal region. Additionally, because of a non-negligible contribution of $\mathrm{W} / \mathrm{Z}+\mathrm{HF}$ events in this sample, the imperfections in the $\mathrm{W} / \mathrm{Z}+$ jets background modeling in the simulation are absorbed into the $\mathrm{t} \overline{\mathrm{t}}$ background prediction. In order to derive the $t \overline{\mathrm{t}}$ shape, we constrain the $\mathrm{W} / \mathrm{Z}+$ jets shape to that obtained from the MC simulation (section 6.1). We find that a two-exponential function provides a good fit to the observed data in the MU box, as shown in figure 3.

\subsection{Multijet background}

The remaining backgrounds that contribute significantly to the interesting region of high $R^{2}$ originate from heavy-flavor enriched multijet production. We use events with a loose muon in the MU box to derive the multijets background PDF. According to the MC simulation, this sample is composed $45 \%$ of top events, $5 \%$ of $\mathrm{W} / \mathrm{Z}+\mathrm{b}$ jet events, and $50 \%$ of multijet events.

We proceed to perform the fits, for which the contributions from $\mathrm{W} / \mathrm{Z}+\mathrm{b}$ jets and $t \overline{\mathrm{t}}$ backgrounds are fixed to the PDFs described in sections 6.1 and 6.2. Based on simulation studies it is found that the parameters of the second component in the fit function $\left(A_{2}\right.$ and $B_{2}$ in eq. (6.1)) are nearly idenical for the multijet and the $t \bar{t}+$ jets background processes. In order to better constrain the multijet fit, the parameters of the second component are set equal to those from the observed events for $t \bar{t}+$ jets while the parameters of the first component of the multijet PDF are left free. The results of the fit in the $2 \mathrm{~b}$-tagged MU box are displayed in figure 4, where we find good agreement between the fit results and observed data. 

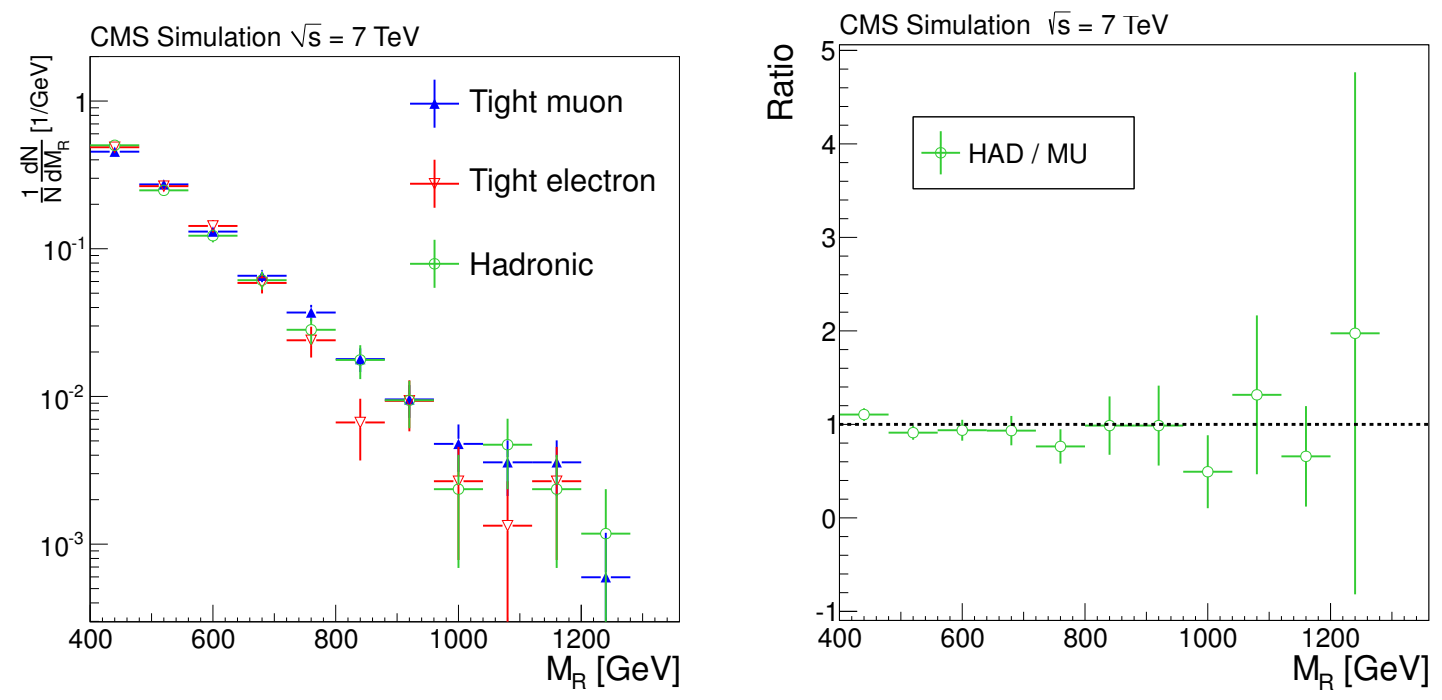

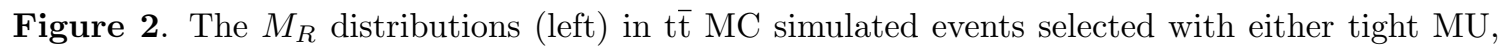
tight ELE and HAD requirements, and (right) the ratio of the number of events selected with the HAD or tight MU selections, as a function of $M_{R}$.
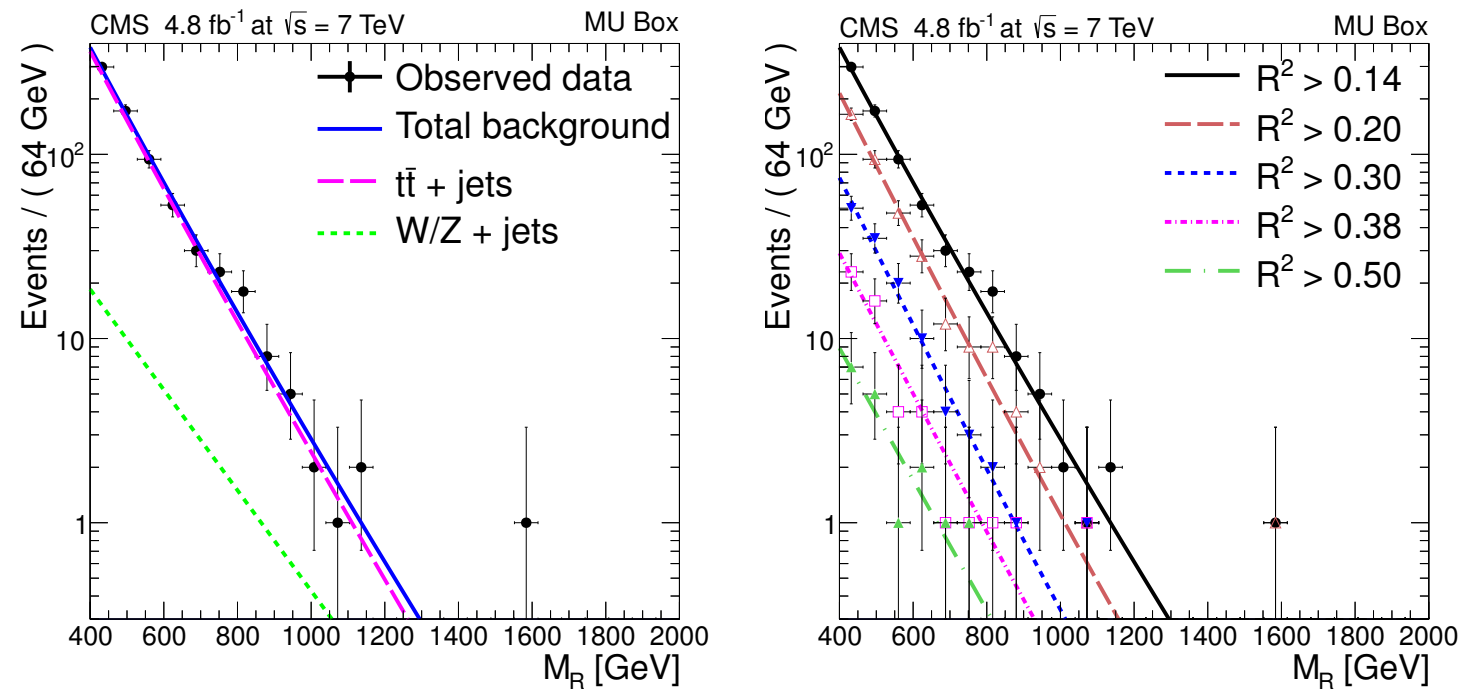

Figure 3. The result of the fit of the $M_{R}$ distributions (lines) compared to MU box observed events with $R^{2}>0.14$ (left); individual background contributions are not stacked. On the right are shown the $M_{R}$ distributions for different values of the $R^{2}$ threshold (right) in 2b-tagged events of the MU box with a tight muon; the results of the fits (lines) are overlaid with the observed distributions (markers). 

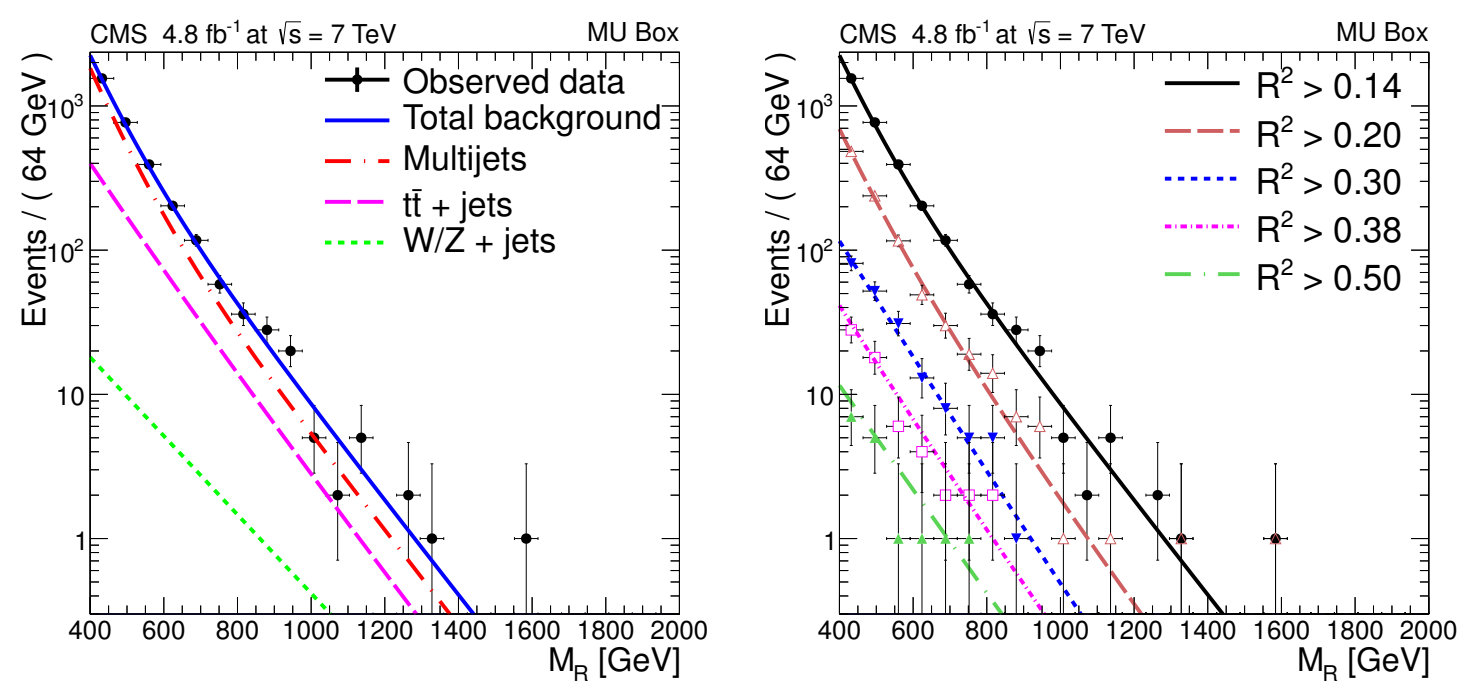

Figure 4. The result of the fit of the $M_{R}$ distributions (lines) compared to the MU box observed data for events with $R^{2}>0.14$ (left); individual contributions of backgrounds are not stacked. On the right are shown the $M_{R}$ distributions for different values of the $R^{2}$ threshold (right) in 2b-tagged events of the MU box with a loose muon; the results of the fits (lines) are overlaid with the observed distributions (markers).

\subsection{Systematic uncertainties}

For the backgrounds estimated from observed events, the uncertainty in the total yield arises from the uncertainties (statistical and systematic) in the fit parameters in eq. (6.1). We estimate these uncertainties by varying the $R^{2}$ threshold values (by $\pm 5 \%$ ), thus arriving at a new set of $A_{i}$ and $B_{i}$ parameters describing the background PDF. The maximum difference observed between the experimental data and the simulated data in the MU box with tight and loose muon selections is then used as the uncertainty on the shape parameters. This procedure results in a $10 \%$ uncertainty in the $A_{i}$ values, and $40 \%$ in the $B_{i}$ values. We also tested the stability of the fits by varying the initial parameters used to start the fit by $\pm 50 \%$, and found that this variation results in stable solutions, returning the same central value for the $A_{i}$ and $B_{i}$ parameters.

We generate an ensemble of pseudoexperiments, based on the fit results in the MU box. From each pseudoexperiment a new set of values for the parameters is then obtained, with the corresponding uncertainties, and we use the associated PDF results to predict the background yield. The ensemble of pseudoexperiments thus provides a distribution of the expected background yield in the signal regions, with its corresponding uncertainty. This procedure allows us to correctly propagate the systematic uncertainty in the background shape into the prediction of the background. To account for the normalization uncertainty we propagate the uncertainty in the $f_{R^{2}}$ introduced in section 5 to the prediction of background yields in the signal region from control samples in observed events. 
The effect of the jet energy scale (JES) and jet energy resolution (JER) uncertainties on the $\mathrm{W} / \mathrm{Z}+$ jets background estimate and the signal model yields from simulation are taken into account. These effects are evaluated by repeating the extraction of all background PDFs by first varying the JES/JER by plus or minus one standard deviation in the $\mathrm{W} / \mathrm{Z}+$ jet background model, and recalculating the $\mathbb{E}_{\mathrm{T}}$ and $R$. These variations correspond to uncertainties as large as $3 \%$ in the selection efficiency. We then re-derive the background model PDFs from observed data in the MU box, using the newly obtained $\mathrm{W} / \mathrm{Z}+\mathrm{HF}$ jets model. The new set of PDFs with their corresponding covariance matrices then serve as an alternative background model.

We apply a scale factor of about 0.95 , that is weakly dependent on jet $p_{\mathrm{T}}$, to account for an observed difference in tagging efficiency between data and simulation. The uncertainty in the scale factor varies from 0.03 to 0.05 for jets with $p_{\mathrm{T}}$ from 30 to $670 \mathrm{GeV}$, and is 0.10 for $\mathrm{b}$ jets with $p_{\mathrm{T}}>670 \mathrm{GeV}$. These uncertainties are measured using a dijet sample with high b-jet purity, as detailed in ref. [33].

The uncertainty in the $\widetilde{b}_{1}$ acceptance due to uncertainties in the parton distribution functions is calculated using the recommendation from the PDF4LHC group [38]. The parton distribution function and the $\alpha_{s}$ variations of next-to-leading (NLO) order in the MSTW2008 [39], CTEQ6.6 [40], and NNPDF2.0 [41] sets were taken into account and their impact on the signal cross sections was compared with the calculation with CTEQ6L1 [42] that was used in the simulation of the signal samples. From these three sets we evaluate an upper and lower bound on the signal efficiency for each pair of assumed $\widetilde{b}_{1}$ and $\widetilde{\chi}_{1}^{0}$ masses, and half of the difference between the two bounds is used as an estimate of the uncertainty. The theoretical cross section of $\mathrm{LQ}_{3}$ production has been calculated using CTEQ6L1 and CTEQ6M [42] at NLO, and the uncertainty in the prediction of the cross section was estimated by repeating the calculation using the NLO MRST2002 parametrization [43]. This uncertainty was found to vary from 3.5 to $25 \%$ for leptoquarks in the mass range considered in this analysis [44].

The systematic uncertainty to the luminosity measurement is taken to be $2.2 \%$ [45], which is correlated among all signal channels and the background estimates that are derived from simulations. The uncertainty in trigger efficiency is estimated using a set of prescaled razor triggers with low thresholds, and is found to be $2 \%$ for events in the HAD box, and $3 \%$ for events in the MU and ELE boxes.

\subsection{ELE control region}

In order to check that our background shape modeling indeed predicts the observed data adequately, we use the PDFs obtained in the steps described above (sections 6.1-6.3) in an orthogonal sample in the $2 \mathrm{~b}$-tagged ELE box with a tight electron selection, i.e. the sample with a well-identified electron, which is then treated as a neutrino. This signal-depleted sample provides an independent cross-check of our background modeling, and covers the same region in $R$ and $M_{R}$ as the HAD box. Additionally, based on MC simulation studies, the composition of the tight ELE sample in observed events is similar to that of the HAD sample, consisting of approximately $85 \% \mathrm{t} \overline{\mathrm{t}}, 5 \% \mathrm{~W} / \mathrm{Z}+\mathrm{HF}$ jets, and $10 \%$ multijet events. 


\begin{tabular}{|ccc|}
\hline & Sideband & Signal-like \\
\hline Multijets & $12.5 \pm 1.9$ & $10 \pm 11$ \\
$\mathrm{~W} / \mathrm{Z}+$ jets & $3.6 \pm 1.9$ & $8.8 \pm 2.8$ \\
$\mathrm{t} \overline{\mathrm{t}}+$ jets & $58.8 \pm 7.7$ & $118.4 \pm 9.8$ \\
Other backgrounds & $0 \pm 0$ & $0.6 \pm 1.0$ \\
\hline$f_{R^{2}, \text { ELE }}$ & \multicolumn{2}{c|}{$0.87 \pm 0.14$} \\
\hline Total background & $65 \pm 13$ & $119 \pm 23$ \\
\hline Observed data & 65 & 121 \\
\hline
\end{tabular}

Table 2. Comparison of the yields in the ELE box. The sideband here refers to $2 \mathrm{~b}$-tagged events in the ELE box with $400<M_{R}<600 \mathrm{GeV}$ and $0.2<R^{2}<0.25$, while "signal-like" refers 2b-tagged events with $M_{R}>400 \mathrm{GeV}$ and $R>0.25$. The scale factor derived in the sideband $\left(f_{R^{2}, \mathrm{ELE}}=0.87 \pm 0.14\right)$ is used to normalize the background yield in the signal-like region (third column), and the uncertainty on the $f_{R^{2}}$, ELE is propagated into the total background yield.

For comparison, the HAD sample is expected to contain approximately $70 \%, 5 \%$, and $25 \%$ of the respective backgrounds.

Using the background model PDFs obtained from the fits, we derive the distribution of the expected shapes in the ELE box using pseudoexperiments. In order to correctly account for correlations and uncertainties in the parameters describing the background model, the shape parameters used to generate each pseudoexperiment data set are sampled from the covariance matrix returned by the fit. The actual number of events in each dataset is then drawn from a Poisson distribution centered on the yield returned by the covariance-matrix sampling. For each pseudoexperiment dataset, the number of events in the sideband and in the high- $R^{2}$ region is found. We then obtain the scale factor $f_{R^{2} \text {, ELE }}=0.87 \pm 0.14$ from the sideband region, which is used to predict the overall yield of background events in the high $R^{2}$ region of the ELE box.

The comparison of the predicted $M_{R}$ distribution with the observed events in the ELE box is shown in figure 5, and the background model is found to predict the observed data adequately. We also test our ability to correctly predict the yields of SM backgrounds using the scale factor mentioned above. The results are summarized in table 2. Total background yield in the sideband is normalized to the number of observed data events in the sideband, in order to derive the scale factor $f_{R^{2} \text {, ELE }}$, as described in section 5 . The uncertainties in the background yields shown here represent systematic uncertainties that are estimated by varying the parameters $A_{i}$ and $B_{i}$, as described in section 6.4 . As can be seen in this comparison, the $f_{R^{2}}$, ELE obtained from the sideband allows one to predict the overall normalization of the $2 \mathrm{~b}$-tagged sample.

We perform another check to test whether the $R^{2}$-dependence is well-described by our background model. This check is needed since in the final signal region we have several signal boxes, each optimized for different signal mass hypotheses. In order to increase the sensitivity for higher masses, a tighter selection on $R^{2}$ is imposed to reduce the backgrounds 


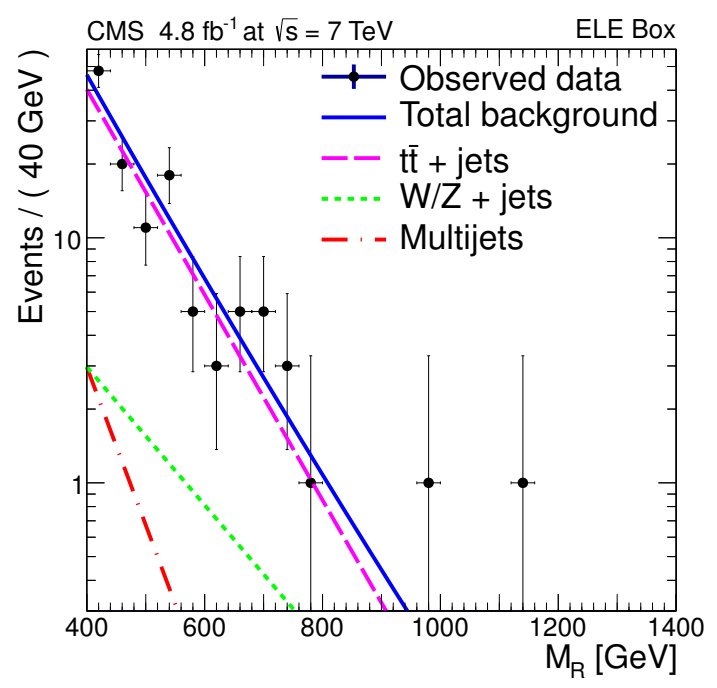

Figure 5. The $M_{R}$ distribution for observed data in the 2b-tagged ELE box for events with $R^{2}>0.25$ compared to the prediction. The background model derived from the MU box is used to predict the $M_{R}$ shapes of the background processes. The individual contributions are not stacked.

\begin{tabular}{|ccc|}
\hline$R^{2}$ Cut & Expected yields & Observed yields \\
\hline$>0.25$ & $119 \pm 23$ & 121 \\
\hline $0.25-0.30$ & $51 \pm 17$ & 48 \\
$0.30-0.35$ & $30 \pm 10$ & 26 \\
$0.35-0.38$ & $9.9 \pm 5.2$ & 11 \\
$0.38-0.42$ & $11.5 \pm 5.0$ & 11 \\
$>0.42$ & $16.8 \pm 4.8$ & 25 \\
\hline
\end{tabular}

Table 3. Expected and observed yields in the 2b-tagged ELE box for $R^{2}$ selections and a fixed requirement $M_{R}>400 \mathrm{GeV}$. The quoted uncertainties on the expected number of events include statistical and systematic uncertainties, and the uncertainty on the scale factor $f_{R^{2}}$, ELE.

further, while keeping the signal efficiency high. In order to ensure that our background model adequately describes observed data with higher $R^{2}$ thresholds, we perform the same procedure in the ELE box. The results are summarized in table 3. Here, we use the same $f_{R^{2} \text {, ELE }}$ derived from the sideband. As can be seen from these results, this model correctly predicts the total yields for higher $R^{2}$ boxes.

\section{Results}

We search for $\mathrm{LQ}_{3}$ and $\widetilde{\mathrm{b}}_{1}$ signals in the HAD box data sample using the background PDFs obtained from the MU box (sections 6.1-6.3). The predicted background yields and their 


\begin{tabular}{|ccc|}
\hline & Sideband & Signal-like \\
\hline Multijets & $81.0 \pm 9.5$ & $34.5 \pm 6.5$ \\
$\mathrm{~W} / \mathrm{Z}+$ jets & $4.5 \pm 2.2$ & $11.4 \pm 3.5$ \\
$\mathrm{t} \overline{\mathrm{t}}+$ jets & $68.7 \pm 9.6$ & $140 \pm 11$ \\
Other backgrounds & $0.08 \pm 0.29$ & $0.16 \pm 0.48$ \\
\hline$f_{R^{2}, \text { HAD }}$ & \multicolumn{2}{c|}{$1.10 \pm 0.13$} \\
\hline Total background & $170 \pm 25$ & $205 \pm 28$ \\
\hline Observed data & 170 & 200 \\
\hline
\end{tabular}

Table 4. Comparison of the yields in the 2b-tagged (signal region) samples in the HAD box. The uncertainties include the systematic uncertainty in the background shapes (section 6.4) and statistical uncertainties. The uncertainty in the total yield after scaling also includes the jet energy scale uncertainty. The scale factor derived in the sideband $\left(f_{R^{2}, \mathrm{HAD}}=1.10 \pm 0.13\right)$ is used to normalize the background yield in the signal-like region. The uncertainty in $f_{R^{2} \text {, HAD }}$ is propagated and included in the quoted uncertainty in the expected background yields.
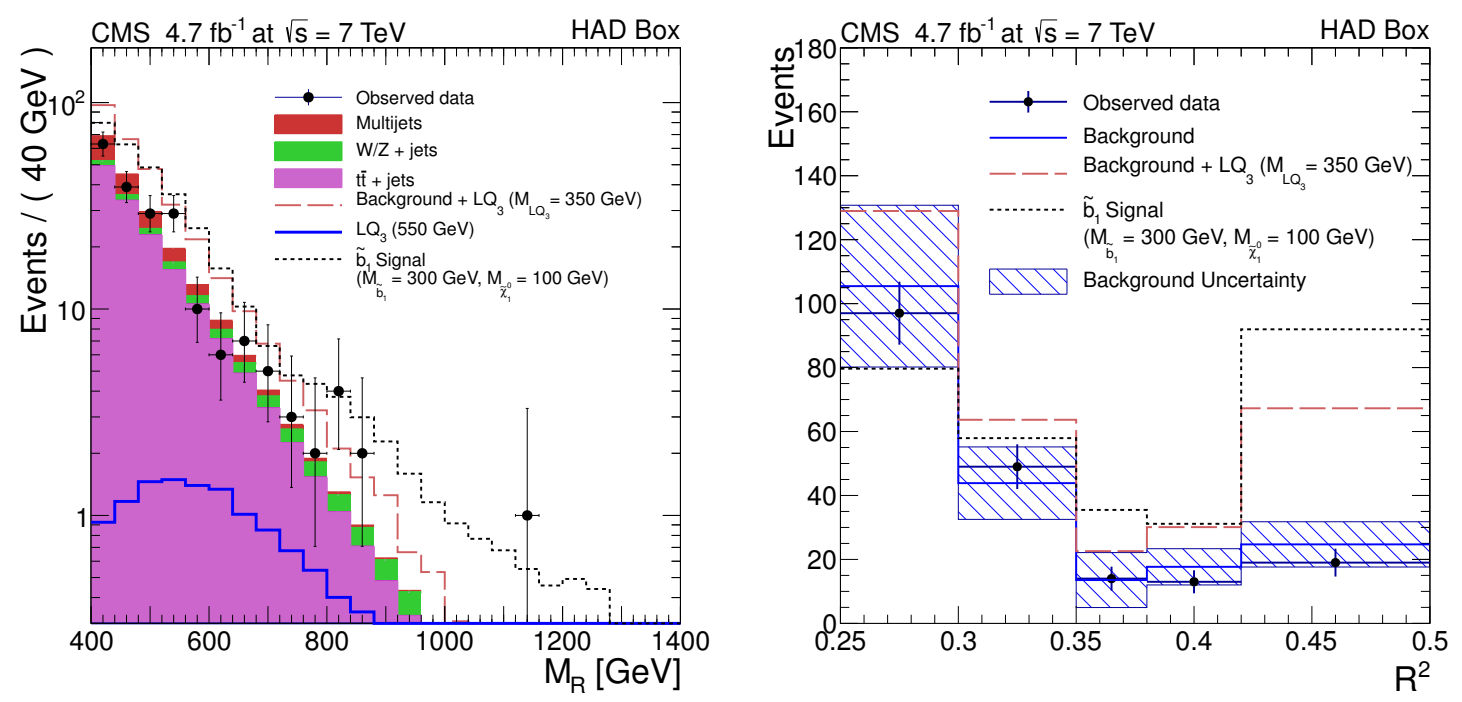

Figure 6. Comparison of the background prediction with the data observed in the $2 \mathrm{~b}$-tagged sample in the HAD signal box for the $M_{R}$ (left) and $R$ (right) distributions. The expected contributions from $\mathrm{LQ}_{3}$ and $\widetilde{\mathrm{b}}_{1}$ signal events with various mass hypotheses are also shown.

uncertainties are summarized in table 4 . Total background yield in the $2 \mathrm{~b}$-tagged sideband is normalized to the number of observed data events in the sideband, in order to derive the scale factor $f_{R^{2}, \mathrm{HAD}}=1.10 \pm 0.13$, as described in section 5. The distributions of $R$ and $M_{R}$ observed in the $2 \mathrm{~b}$-tagged HAD box are compared to the background prediction in figure 6 .

As seen in figure 6 and table 4 , both the number of observed events and the shapes of the $R$ and $M_{R}$ distributions are in agreement with the expected SM backgrounds. Therefore, 


\begin{tabular}{|cccccc|}
\hline$R^{2}$ Cut & Expected yields & Observed yields & $R^{2}$ bins & Expected yields & Observed yields \\
\hline$>0.25$ & $205 \pm 28$ & 200 & $0.25-0.30$ & $105 \pm 25$ & 97 \\
$>0.30$ & $100 \pm 16$ & 103 & $0.30-0.35$ & $44 \pm 11$ & 49 \\
$>0.35$ & $56 \pm 12$ & 54 & $0.35-0.38$ & $13 \pm 9$ & 14 \\
$>0.38$ & $43 \pm 9$ & 40 & $0.38-0.42$ & $18 \pm 6$ & 13 \\
$>0.42$ & $25 \pm 7$ & 27 & $>0.42$ & $25 \pm 7$ & 27 \\
\hline
\end{tabular}

Table 5. Expected and observed yields in the 2b-tagged HAD box for various $R^{2}$ selections and a fixed $M_{R}>400 \mathrm{GeV}$ requirement. The quoted uncertainties on the expected number of events include statistical and systematic uncertainties, and the uncertainty from the $f_{R^{2} \text {, HAD }}$ The left three columns show inclusive yields above the $R^{2}$ threshold, while the right three columns show the yields in bins of $R^{2}$.

we proceed to define two signal regions, to enhance the sensitivity for different $\mathrm{LQ}_{3}$ masses. The regions are optimized to provide the lowest expected cross section limits, by varying the thresholds on $R$ and $M_{R}$. We find that $M_{R}>400 \mathrm{GeV}$ provides the best sensitivity for all masses, and for $\mathrm{LQ}_{3}$ masses below $350 \mathrm{GeV}$ the optimal selection is $R^{2}>0.25$, while for higher masses $R^{2}>0.42$ provides best sensitivity. Because of the high value assumed for the $\widetilde{\chi}_{1}^{0}$ mass in the $\widetilde{\mathrm{b}}_{1}$ search, the inclusive selection of $M_{R}>400 \mathrm{GeV}$ and $R^{2}>0.25$ is found to provide the optimal sensitivity in the mass range considered in this analysis.

Table 5 shows the comparison of the expected background yields in these signal boxes, and agreement of the observed event counts with the expectations is observed. Table 6 shows the efficiency of these selections for several $\mathrm{LQ}_{3}$ mass hypotheses, based on $\mathrm{MC}$ simulation. Efficiencies for the $\widetilde{b}_{1}$ signal are shown in figure 7 . Typical efficiencies range from a few percent up to $\sim 12$ percent for $\widetilde{b}_{1}$ masses between 200 and $500 \mathrm{GeV}$ and small $\widetilde{\chi}_{1}^{0}$ mass. The efficiency drops when the mass of the $\widetilde{b}_{1}$ squark is close to the mass of $\widetilde{\chi}_{1}^{0}$, since the resulting $\mathrm{b}$ jets are softer in these scenarios.

The statistical model for the observed number of events is a Poisson distribution with the expected value equal to the sum of the signal and expected backgrounds. Log-normal priors for the nuisance parameters are used to model the systematic uncertainties listed in section 6.4.

A $95 \%$ CL upper limit is set on the potential signal cross section, as summarized in table 7. The modified frequentist construction $\mathrm{CL}_{\mathrm{s}}[46,47]$ is used for limit calculation. These limits are interpreted in terms of limits of $\mathrm{LQ}_{3}$ pair production cross section as shown in figure 8. The upper limits are compared to the NLO prediction of the LQ pair production cross section [44], and we set a 95\% CL exclusion on LQ masses smaller than $440 \mathrm{GeV}$ (expected $470 \mathrm{GeV}$ ), assuming $\beta=0$. We also present the $95 \% \mathrm{CL}$ limit on $\beta$ as a function of $\mathrm{LQ}_{3}$ mass as shown on the right side of figure 8 .

The results of the analysis are interpreted in the context of the simplified supersymmetry model spectra (SMS) [48-50]. In SMS, a limited set of hypothetical particles and decay chains are introduced to produce a given topological signature, such as the $\mathbb{E}_{\mathrm{T}}$ plus 


\begin{tabular}{|cccc|}
\hline$M_{\mathrm{LQ}_{3}}[\mathrm{GeV}]$ & $\sigma(\mathrm{pb})$ & Efficiency $(\%)$ & $\begin{array}{c}\text { Number } \\
\text { of expected events }\end{array}$ \\
\hline 200 & 12 & 0.33 & $185 \pm 13$ \\
250 & 3.5 & 1.1 & $171.2 \pm 9.1$ \\
280 & 1.8 & 1.8 & $151.4 \pm 3.6$ \\
320 & 0.82 & 3.2 & $122.8 \pm 1.9$ \\
350 & 0.48 & 1.8 & $39.2 \pm 1.3$ \\
450 & 0.095 & 4.3 & $19.17 \pm 0.38$ \\
550 & 0.024 & 5.9 & $6.59 \pm 0.12$ \\
\hline
\end{tabular}

Table 6. Summary of the expected $\mathrm{LQ}_{3}$ signal yields and efficiency in the signal region, for $4.7 \mathrm{fb}^{-1}$ of observed data, in events with $M_{R}>400 \mathrm{GeV}$. For LQ $\mathrm{LQ}_{3}$ masses below $350 \mathrm{GeV} R^{2}>0.25$ is required, while for heavier masses we require events to pass $R^{2}>0.42$. All uncertainties are statistical only.

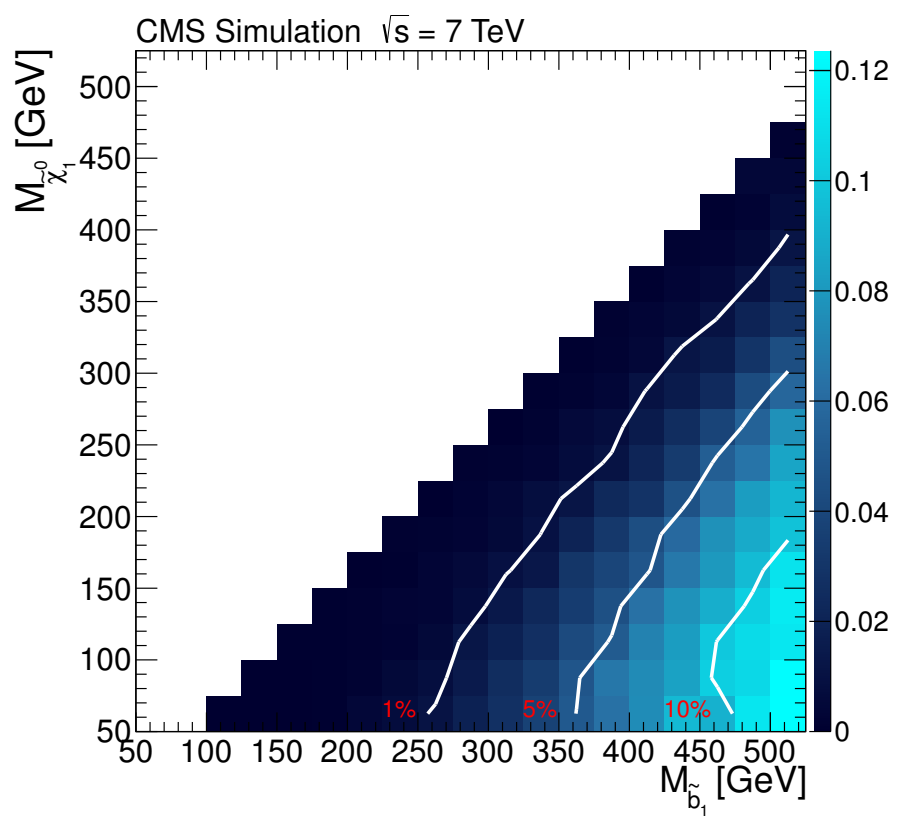

Figure 7. Signal efficiency for simulated $\widetilde{\mathrm{b}}_{1}$ signal events with $M_{R}>400 \mathrm{GeV}$ and $R^{2}>0.25$. White lines show the iso-efficiency contours for 1,5 , and $10 \%$ signal efficiency, respectively.

$\mathrm{b}$ jets final state considered in this analysis. We consider a SMS scenario where all supersymmetric particles are set to have a very large mass, except for the $\widetilde{b}_{1}$ and $\widetilde{\chi}_{1}^{0}$. The pairs of scalar bottom quarks produced through strong interactions are kinematically allowed to decay only into a b quark and a $\widetilde{\chi}_{1}^{0}$.

The observed and expected $95 \%$ CL upper limits in the $\tilde{b}_{1}-\widetilde{\chi}_{1}^{0}$ mass plane are shown in figure 9 , where the $\widetilde{b}_{1}$ pair production cross section is calculated at the NLO and next-to- 


\begin{tabular}{|ccccccc|}
\hline$M_{\mathrm{LQ}_{3}}[\mathrm{GeV}]$ & $-2 \sigma$ & $-1 \sigma$ & $\begin{array}{c}\text { Median } \\
\text { expected limit }[\mathrm{pb}]\end{array}$ & $+1 \sigma$ & $+2 \sigma$ & $\begin{array}{c}\text { Observed } \\
\text { limit }[\mathrm{pb}]\end{array}$ \\
\hline 200 & 2.0 & 3.3 & 4.5 & 6.2 & 8.4 & 4.3 \\
250 & 0.64 & 1.1 & 1.4 & 2.0 & 2.6 & 1.3 \\
270 & 0.43 & 0.75 & 0.97 & 1.4 & 1.8 & 0.90 \\
330 & 0.18 & 0.24 & 0.33 & 0.46 & 0.62 & 0.36 \\
350 & 0.13 & 0.17 & 0.23 & 0.32 & 0.42 & 0.25 \\
450 & 0.047 & 0.067 & 0.092 & 0.13 & 0.17 & 0.10 \\
550 & 0.037 & 0.049 & 0.066 & 0.094 & 0.13 & 0.073 \\
\hline
\end{tabular}

Table 7. Observed and expected 95\% CL upper limits on the $\mathrm{LQ}_{3}$ pair-production cross section as a function of the $\mathrm{LQ}_{3}$ mass.
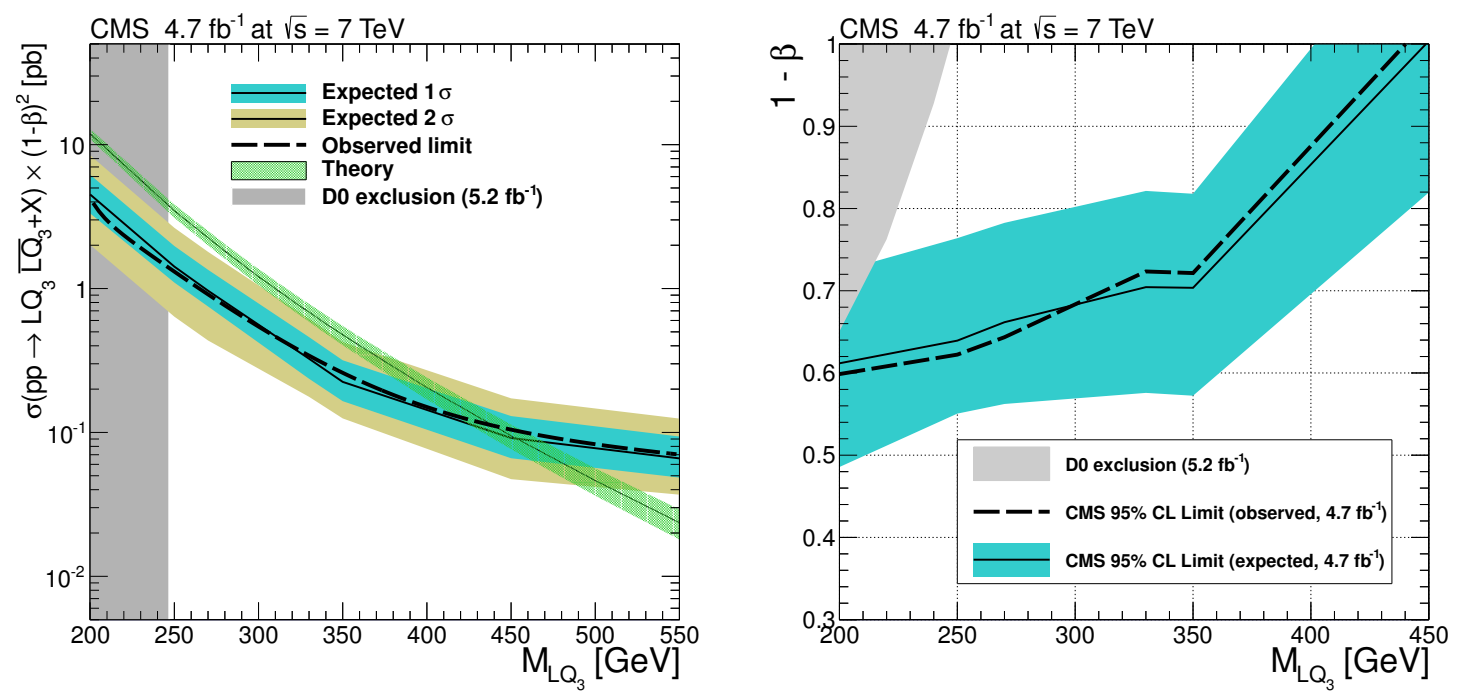

Figure 8. (Left) the expected and observed upper limit at 95\% CL on the $\mathrm{LQ}_{3}$ pair production cross section as a function of the $\mathrm{LQ}_{3}$ mass, assuming $\beta=0$. The systematic uncertainties reported in section 6.4 are included in the calculation. The vertical greyed region is excluded by the current D0 limit [12] in the same channel. The theory curve and its band represent, respectively, the theoretical $\mathrm{LQ}_{3}$ pair production cross section and the uncertainties due to the choice of parton distribution functions and renormalization/factorization scales [44]. (Right) minimum $\beta$ for a $95 \%$ $\mathrm{CL}$ exclusion of the $\mathrm{LQ}_{3}$ hypothesis as a function of $\mathrm{LQ}_{3}$ mass. The observed (expected) exclusion curve is obtained using the observed (expected) upper limit and the central value of the theoretical $\mathrm{LQ}_{3}$ pair production cross section. The band around the observed exclusion curve is obtained by considering the observed upper limit while taking into account the uncertainties on the theoretical cross section. The grey region is excluded by the current D0 limits [12] in the same channel. 


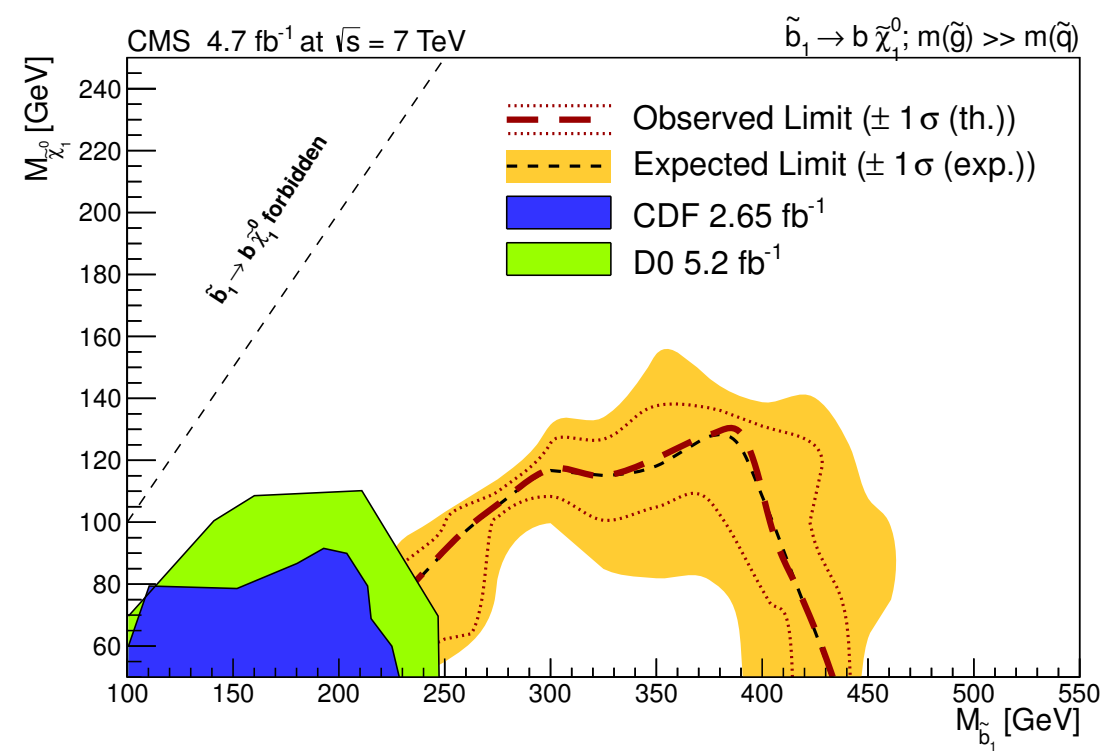

Figure 9. The expected and observed $95 \%$ CL exclusion limits for the $\widetilde{b}_{1}$ pair production SMS model. The red dashed contour shows the 95\% CL exclusion limits based on the NLO+NLL cross section. The red dotted contours represent the theoretical uncertainties from the variation of parton distribution functions, and renormalization and factorization scales. The corresponding expected limits are shown with the black dashed contour. The shaded yellow contours represent the uncertainties in the SM background estimates, as reported in section 6.4.

leading-logarithm (NLL) order [51-56]. Since $M_{R}$ depends on the squared difference of the masses of $\widetilde{\mathrm{b}}_{1}$ and $\widetilde{\chi}_{1}^{0}$, at the $\widetilde{\mathrm{b}}_{1}$ masses around $400-450 \mathrm{GeV}$ and low $\widetilde{\chi}_{1}^{0}$ masses the exclusion limit is almost independent of the $\widetilde{\chi}_{1}^{0}$ mass. The signal acceptance in the region with small mass splitting between the $\widetilde{\mathrm{b}}_{1}$ and $\widetilde{\chi}_{1}^{0}$ is particularly susceptible to uncertainties associated with initial-state radiation (ISR). The impact of ISR is estimated by comparing the results of the acceptance calculation using PYTHIA with the "power shower" and with moderate ISR settings [19]. If the acceptance varies by more than $25 \%$ for a particular choice of $\widetilde{b}_{1}$ and $\widetilde{\chi}_{1}^{0}$ masses, then no limit is set for those mass parameters. This procedure results in reduced sensitivity in the region of $m\left(\widetilde{\mathrm{b}}_{1}\right)<300 \mathrm{GeV}$ and $80<m\left(\widetilde{\chi}_{1}^{0}\right)<130 \mathrm{GeV}$, and thus an inability to exclude some of the models in this parameter range.

\section{Summary}

A search has been performed for third-generation scalar leptoquarks and for scalar bottom quarks in the all-hadronic channel with a signature of large $\mathbb{E}_{\mathrm{T}}$ and b-tagged jets. This search is based on a data sample collected in pp collisions at $\sqrt{s}=7 \mathrm{TeV}$ and corresponding to an integrated luminosity of $4.7 \mathrm{fb}^{-1}$. The number of observed events is in agreement with the predictions for the SM backgrounds. We set an upper limit on the $\mathrm{LQ}_{3}$ pair production cross section, excluding a scalar $\mathrm{LQ}_{3}$ with mass below $450 \mathrm{GeV}$, assuming a $100 \%$ branching fraction of the $\mathrm{LQ}_{3}$ to b quarks and tau neutrinos. We set $95 \%$ confidence level upper limits 
in the $\tilde{b}_{1}-\widetilde{\chi}_{1}^{0}$ mass plane such that for neutralino masses of $50 \mathrm{GeV}$, scalar bottom masses up to $410 \mathrm{GeV}$ are excluded. These results represent the most stringent limits on $\mathrm{LQ}_{3}$ masses and extend limits on $\widetilde{\mathrm{b}}_{1}$ masses to much higher values than probed previously.

\section{Acknowledgments}

We congratulate our colleagues in the CERN accelerator departments for the excellent performance of the LHC machine. We thank the technical and administrative staff at CERN and other CMS institutes, and acknowledge support from BMWF and FWF (Austria); FNRS and FWO (Belgium); CNPq, CAPES, FAPERJ, and FAPESP (Brazil); MEYS (Bulgaria); CERN; CAS, MoST, and NSFC (China); COLCIENCIAS (Colombia); MSES (Croatia); RPF (Cyprus); MoER, SF0690030s09 and ERDF (Estonia); Academy of Finland, MEC, and HIP (Finland); CEA and CNRS/IN2P3 (France); BMBF, DFG, and HGF (Germany); GSRT (Greece); OTKA and NKTH (Hungary); DAE and DST (India); IPM (Iran); SFI (Ireland); INFN (Italy); NRF and WCU (Korea); LAS (Lithuania); CINVESTAV, CONACYT, SEP, and UASLP-FAI (Mexico); MSI (New Zealand); PAEC (Pakistan); MSHE and NSC (Poland); FCT (Portugal); JINR (Armenia, Belarus, Georgia, Ukraine, Uzbekistan); MON, RosAtom, RAS and RFBR (Russia); MSTD (Serbia); SEIDI and CPAN (Spain); Swiss Funding Agencies (Switzerland); NSC (Taipei); ThEP, IPST and NECTEC (Thailand); TUBITAK and TAEK (Turkey); NASU (Ukraine); STFC (United Kingdom); DOE and NSF (U.S.A.). Individuals have received support from the Marie-Curie programme and the European Research Council (European Union); the Leventis Foundation; the A. P. Sloan Foundation; the Alexander von Humboldt Foundation; the Belgian Federal Science Policy Office; the Fonds pour la Formation à la Recherche dans l'Industrie et dans l'Agriculture (FRIA-Belgium); the Agentschap voor Innovatie door Wetenschap en Technologie (IWT-Belgium); the Ministry of Education, Youth and Sports (MEYS) of Czech Republic; the Council of Science and Industrial Research, India; the Compagnia di San Paolo (Torino); and the HOMING PLUS programme of Foundation for Polish Science, cofinanced from European Union, Regional Development Fund.

Open Access. This article is distributed under the terms of the Creative Commons Attribution License which permits any use, distribution and reproduction in any medium, provided the original author(s) and source are credited.

\section{References}

[1] J.C. Pati and A. Salam, Lepton number as the fourth color, Phys. Rev. D 10 (1974) 275 [Erratum ibid. D 11 (1975) 703] [INSPIRE].

[2] B. Schrempp and F. Schrempp, Light leptoquarks, Phys. Lett. B 153 (1985) 101 [inSPIRE].

[3] B. Gripaios, A. Papaefstathiou, K. Sakurai and B. Webber, Searching for third-generation composite leptoquarks at the LHC, JHEP 01 (2011) 156 [arXiv:1010.3962] [INSPIRE].

[4] S. Dimopoulos and L. Susskind, Mass without scalars, Nucl. Phys. B 155 (1979) 237 [INSPIRE]. 
[5] S. Dimopoulos, Technicolored signatures, Nucl. Phys. B 168 (1980) 69 [InSPIRE].

[6] E. Farhi and L. Susskind, Technicolor, Phys. Rept. 74 (1981) 277 [InSPIRE].

[7] J.L. Hewett and T.G. Rizzo, Low-energy phenomenology of superstring inspired $E_{6}$ models, Phys. Rept. 183 (1989) 193 [inSPIRE].

[8] S. Davidson, D.C. Bailey and B.A. Campbell, Model independent constraints on leptoquarks from rare processes, Z. Phys. C 61 (1994) 613 [hep-ph/9309310] [InSPIRE].

[9] G.R. Farrar and P. Fayet, Phenomenology of the production, decay and detection of new hadronic states associated with supersymmetry, Phys. Lett. B 76 (1978) 575 [INSPIRE].

[10] J.L. Feng, Dark matter candidates from particle physics and methods of detection, Ann. Rev. Astron. Astrophys. 48 (2010) 495 [arXiv: 1003.0904] [INSPIRE].

[11] S. Dimopoulos and G. Giudice, Naturalness constraints in supersymmetric theories with nonuniversal soft terms, Phys. Lett. B 357 (1995) 573 [hep-ph/9507282] [INSPIRE].

[12] D0 collaboration, V.M. Abazov et al., Search for scalar bottom quarks and third-generation leptoquarks in p $\bar{p}$ collisions at $\sqrt{s}=1.96$ TeV, Phys. Lett. B 693 (2010) 95 [arXiv: 1005.2222] [INSPIRE].

[13] CDF collaboration, T. Aaltonen et al., Search for the production of scalar bottom quarks in $p \bar{p}$ collisions at $\sqrt{s}=1.96$ TeV, Phys. Rev. Lett. 105 (2010) 081802 [arXiv:1005.3600] [INSPIRE].

[14] CMS collaboration, Search for pair production of third-generation leptoquarks and top squarks in pp collisions at $\sqrt{s}=7 \mathrm{TeV}$, arXiv:1210.5629 [INSPIRE].

[15] ATLAS collaboration, Search for scalar bottom pair production with the ATLAS detector in pp collisions at $\sqrt{s}=7 \mathrm{TeV}$, Phys. Rev. Lett. 108 (2012) 181802 [arXiv:1112.3832] [INSPIRE].

[16] CMS collaboration, The CMS experiment at the CERN LHC, 2008 JINST 3 S08004 [INSPIRE].

[17] C. Rogan, Kinematical variables towards new dynamics at the LHC, arXiv:1006.2727 [INSPIRE].

[18] CMS collaboration, Inclusive search for squarks and gluinos in pp collisions at $\sqrt{s}=7 \mathrm{TeV}$, Phys. Rev. D 85 (2012) 012004 [arXiv:1107.1279] [InSPIRE].

[19] T. Sjöstrand, S. Mrenna and P.Z. Skands, PYTHIA 6.4 physics and manual, JHEP 05 (2006) 026 [hep-ph/0603175] [INSPIRE].

[20] J. Alwall, M. Herquet, F. Maltoni, O. Mattelaer and T. Stelzer, MadGraph 5: going beyond, JHEP 06 (2011) 128 [arXiv:1106.0522] [InSPIRE].

[21] GEANT4 collaboration, S. Agostinelli et al., GEANT4: a simulation toolkit, Nucl. Instrum. Meth. A 506 (2003) 250 [INSPIRE].

[22] CMS collaboration, Measurement of the underlying event activity at the LHC with $\sqrt{s}=7 \mathrm{TeV}$ and comparison with $\sqrt{s}=0.9 \mathrm{TeV}$, JHEP 09 (2011) 109 [arXiv:1107.0330] [INSPIRE].

[23] R. Field, Physics at the Tevatron, Acta Phys. Pol. B 39 (2008) 2611 [InSPIRE]. 
[24] R. Field, Studying the underlying event at CDF and the LHC, in Multiple partonic interactions at the LHC, proceedings of $1^{\text {st }}$ International Workshop, MPI'08, P. Bartalini and L. Fanó eds., Perugia Italy October 27-31 2008, pg. 12 [arXiv:1003.4220] [INSPIRE].

[25] CMS collaboration, Comparison of the fast simulation of CMS with the first LHC data, CMS-DP-2010-039, CERN, Geneva Switzerland (2010).

[26] CMS collaboration, Tracking and primary vertex results in first $7 \mathrm{TeV}$ collisions, CMS-PAS-TRK-10-005, CERN, Geneva Switzerland (2010).

[27] CMS collaboration, Missing transverse energy performance of the CMS detector, 2011 JINST 6 P09001 [arXiv:1106.5048] [INSPIRE].

[28] M. Cacciari, G.P. Salam and G. Soyez, The anti-k $k_{t}$ jet clustering algorithm, JHEP 04 (2008) 063 [arXiv:0802.1189] [INSPIRE].

[29] CMS collaboration, Determination of jet energy calibration and transverse momentum resolution in CMS, 2011 JINST 6 P11002 [arXiv:1107.4277] [INSPIRE].

[30] CMS collaboration, Commissioning of the particle-flow reconstruction in minimum-bias and jet events from pp collisions at $7 \mathrm{TeV}$, CMS-PAS-PFT-10-002, CERN, Geneva Switzerland (2010).

[31] CMS collaboration, Measurements of inclusive $W$ and $Z$ cross sections in pp collisions at $\sqrt{s}=7 \mathrm{TeV}$, JHEP 01 (2011) 080 [arXiv:1012.2466] [INSPIRE].

[32] M. Cacciari and G.P. Salam, Pileup subtraction using jet areas, Phys. Lett. B 659 (2008) 119 [arXiv: 0707.1378] [INSPIRE].

[33] CMS collaboration, b-jet identification in the CMS experiment, CMS-PAS-BTV-11-004, CERN, Geneva Switzerland (2011).

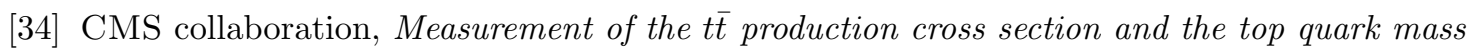
in the dilepton channel in pp collisions at $\sqrt{s}=7$ TeV, JHEP 07 (2011) 049 [arXiv: 1105.5661] [INSPIRE].

[35] W. Verkerke and D.P. Kirkby, The RooFit toolkit for data modeling, eConf C 0303241 (2003) MOLT007 [physics/0306116] [INSPIRE].

[36] CMS collaboration, Study of the dijet invariant mass distribution in $W \rightarrow \ell \nu$ plus jets events produced in pp collisions at $\sqrt{s}=7 \mathrm{TeV}$, CMS-PAS-EWK-11-017, CERN, Geneva Switzerland (2011).

[37] CMS collaboration, Measurement of the $Z / \gamma^{*}+$ b-jet cross section in pp collisions at $7 \mathrm{TeV}$, JHEP 06 (2012) 126 [arXiv:1204.1643] [INSPIRE].

[38] M. Botje et al., The PDF4LHC working group interim recommendations, arXiv:1101.0538 [INSPIRE].

[39] A. Martin, W. Stirling, R. Thorne and G. Watt, Parton distributions for the LHC, Eur. Phys. J. C 63 (2009) 189 [arXiv:0901.0002] [InSPIRE].

[40] P.M. Nadolsky et al., Implications of CTEQ global analysis for collider observables, Phys. Rev. D 78 (2008) 013004 [arXiv:0802.0007] [INSPIRE].

[41] R.D. Ball et al., A first unbiased global NLO determination of parton distributions and their uncertainties, Nucl. Phys. B 838 (2010) 136 [arXiv:1002.4407] [INSPIRE].

[42] J. Pumplin et al., New generation of parton distributions with uncertainties from global QCD analysis, JHEP 07 (2002) 012 [hep-ph/0201195] [INSPIRE]. 
[43] A. Martin, R. Roberts, W. Stirling and R. Thorne, Uncertainties of predictions from parton distributions. 1: experimental errors, Eur. Phys. J. C 28 (2003) 455 [hep-ph/0211080] [INSPIRE].

[44] M. Krämer, T. Plehn, M. Spira and P. Zerwas, Pair production of scalar leptoquarks at the CERN LHC, Phys. Rev. D 71 (2005) 057503 [hep-ph/0411038] [InSPIRE].

[45] CMS collaboration, Absolute calibration of the luminosity measurement at CMS: winter 2012 update, CMS-PAS-SMP-12-008, CERN, Geneva Switzerland (2012).

[46] A.L. Read, Presentation of search results: the $C L_{s}$ technique, J. Phys. G 28 (2002) 2693 [INSPIRE].

[47] T. Junk, Confidence level computation for combining searches with small statistics, Nucl. Instrum. Meth. A 434 (1999) 435 [hep-ex/9902006] [INSPIRE].

[48] LHC New Physics Working Group collaboration, D. Alves et al., Simplified models for LHC new physics searches, J. Phys. G 39 (2012) 105005 [arXiv:1105.2838] [InSPIRE].

[49] J. Alwall, P. Schuster and N. Toro, Simplified models for a first characterization of new physics at the LHC, Phys. Rev. D 79 (2009) 075020 [arXiv:0810.3921] [INSPIRE].

[50] J. Alwall, M.-P. Le, M. Lisanti and J.G. Wacker, Model-independent jets plus missing energy searches, Phys. Rev. D 79 (2009) 015005 [arXiv: 0809.3264] [InSPIRE].

[51] W. Beenakker, R. Hopker, M. Spira and P. Zerwas, Squark and gluino production at hadron colliders, Nucl. Phys. B 492 (1997) 51 [hep-ph/9610490] [InSPIRE].

[52] A. Kulesza and L. Motyka, Threshold resummation for squark-antisquark and gluino-pair production at the LHC, Phys. Rev. Lett. 102 (2009) 111802 [arXiv:0807.2405] [INSPIRE].

[53] A. Kulesza and L. Motyka, Soft gluon resummation for the production of gluino-gluino and squark-antisquark pairs at the LHC, Phys. Rev. D 80 (2009) 095004 [arXiv: 0905.4749] [INSPIRE].

[54] W. Beenakker et al., Soft-gluon resummation for squark and gluino hadroproduction, JHEP 12 (2009) 041 [arXiv: 0909.4418] [INSPIRE].

[55] W. Beenakker et al., Squark and gluino hadroproduction, Int. J. Mod. Phys. A 26 (2011) 2637 [arXiv: 1105.1110] [INSPIRE].

[56] M. Krämer et al., Supersymmetry production cross sections in pp collisions at $\sqrt{s}=7 \mathrm{TeV}$, arXiv:1206.2892 [INSPIRE]. 


\section{The CMS collaboration}

\section{Yerevan Physics Institute, Yerevan, Armenia}

S. Chatrchyan, V. Khachatryan, A.M. Sirunyan, A. Tumasyan

\section{Institut für Hochenergiephysik der OeAW, Wien, Austria}

W. Adam, E. Aguilo, T. Bergauer, M. Dragicevic, J. Erö, C. Fabjan ${ }^{1}$, M. Friedl, R. Frühwirth ${ }^{1}$, V.M. Ghete, N. Hörmann, J. Hrubec, M. Jeitler ${ }^{1}$, W. Kiesenhofer, V. Knünz, M. Krammer ${ }^{1}$, I. Krätschmer, D. Liko, I. Mikulec, M. Pernicka ${ }^{\dagger}$, D. Rabady ${ }^{2}$, B. Rahbaran, C. Rohringer, H. Rohringer, R. Schöfbeck, J. Strauss, A. Taurok, W. Waltenberger, C.-E. Wulz ${ }^{1}$

National Centre for Particle and High Energy Physics, Minsk, Belarus

V. Mossolov, N. Shumeiko, J. Suarez Gonzalez

\section{Universiteit Antwerpen, Antwerpen, Belgium}

M. Bansal, S. Bansal, T. Cornelis, E.A. De Wolf, X. Janssen, S. Luyckx, L. Mucibello, S. Ochesanu, B. Roland, R. Rougny, M. Selvaggi, H. Van Haevermaet, P. Van Mechelen, N. Van Remortel, A. Van Spilbeeck

\section{Vrije Universiteit Brussel, Brussel, Belgium}

F. Blekman, S. Blyweert, J. D’Hondt, R. Gonzalez Suarez, A. Kalogeropoulos, M. Maes, A. Olbrechts, S. Tavernier, W. Van Doninck, P. Van Mulders, G.P. Van Onsem, I. Villella

\section{Université Libre de Bruxelles, Bruxelles, Belgium}

B. Clerbaux, G. De Lentdecker, V. Dero, A.P.R. Gay, T. Hreus, A. Léonard, P.E. Marage, A. Mohammadi, T. Reis, L. Thomas, C. Vander Velde, P. Vanlaer, J. Wang

\section{Ghent University, Ghent, Belgium}

V. Adler, K. Beernaert, A. Cimmino, S. Costantini, G. Garcia, M. Grunewald, B. Klein, J. Lellouch, A. Marinov, J. Mccartin, A.A. Ocampo Rios, D. Ryckbosch, M. Sigamani, N. Strobbe, F. Thyssen, M. Tytgat, S. Walsh, E. Yazgan, N. Zaganidis

Université Catholique de Louvain, Louvain-la-Neuve, Belgium

S. Basegmez, G. Bruno, R. Castello, L. Ceard, C. Delaere, T. du Pree, D. Favart, L. Forthomme, A. Giammanco ${ }^{3}$, J. Hollar, V. Lemaitre, J. Liao, O. Militaru, C. Nuttens, D. Pagano, A. Pin, K. Piotrzkowski, J.M. Vizan Garcia

\section{Université de Mons, Mons, Belgium}

N. Beliy, T. Caebergs, E. Daubie, G.H. Hammad

\section{Centro Brasileiro de Pesquisas Fisicas, Rio de Janeiro, Brazil}

G.A. Alves, M. Correa Martins Junior, T. Martins, M.E. Pol, M.H.G. Souza

\section{Universidade do Estado do Rio de Janeiro, Rio de Janeiro, Brazil}

W.L. Aldá Júnior, W. Carvalho, A. Custódio, E.M. Da Costa, D. De Jesus Damiao, C. De Oliveira Martins, S. Fonseca De Souza, H. Malbouisson, M. Malek, D. Matos Figueiredo, L. Mundim, H. Nogima, W.L. Prado Da Silva, A. Santoro, L. Soares Jorge, A. Sznajder, A. Vilela Pereira 
Instituto de Fisica Teorica, Universidade Estadual Paulista, Sao Paulo, Brazil T.S. Anjos ${ }^{4}$, C.A. Bernardes ${ }^{4}$, F.A. Dias ${ }^{5}$, T.R. Fernandez Perez Tomei, E.M. Gregores ${ }^{4}$, C. Lagana, F. Marinho, P.G. Mercadante ${ }^{4}$, S.F. Novaes, Sandra S. Padula

Institute for Nuclear Research and Nuclear Energy, Sofia, Bulgaria

V. Genchev ${ }^{2}$, P. Iaydjiev², S. Piperov, M. Rodozov, S. Stoykova, G. Sultanov, V. Tcholakov, R. Trayanov, M. Vutova

University of Sofia, Sofia, Bulgaria

A. Dimitrov, R. Hadjiiska, V. Kozhuharov, L. Litov, B. Pavlov, P. Petkov

Institute of High Energy Physics, Beijing, China

J.G. Bian, G.M. Chen, H.S. Chen, C.H. Jiang, D. Liang, S. Liang, X. Meng, J. Tao, J. Wang, X. Wang, Z. Wang, H. Xiao, M. Xu, J. Zang, Z. Zhang

State Key Lab. of Nucl. Phys. and Tech., Peking University, Beijing, China

C. Asawatangtrakuldee, Y. Ban, Y. Guo, W. Li, S. Liu, Y. Mao, S.J. Qian, H. Teng, D. Wang, L. Zhang, W. Zou

Universidad de Los Andes, Bogota, Colombia

C. Avila, C.A. Carrillo Montoya, J.P. Gomez, B. Gomez Moreno, A.F. Osorio Oliveros, J.C. Sanabria

Technical University of Split, Split, Croatia

N. Godinovic, D. Lelas, R. Plestina ${ }^{6}$, D. Polic, I. Puljak ${ }^{2}$

University of Split, Split, Croatia

Z. Antunovic, M. Kovac

Institute Rudjer Boskovic, Zagreb, Croatia

V. Brigljevic, S. Duric, K. Kadija, J. Luetic, D. Mekterovic, S. Morovic

University of Cyprus, Nicosia, Cyprus

A. Attikis, M. Galanti, G. Mavromanolakis, J. Mousa, C. Nicolaou, F. Ptochos, P.A. Razis

Charles University, Prague, Czech Republic

M. Finger, M. Finger Jr.

Academy of Scientific Research and Technology of the Arab Republic of Egypt, Egyptian Network of High Energy Physics, Cairo, Egypt

Y. Assran ${ }^{7}$, S. Elgammal ${ }^{8}$, A. Ellithi Kamel ${ }^{9}$, A.M. Kuotb Awad ${ }^{10}$, M.A. Mahmoud ${ }^{10}$, A. Radi ${ }^{11,12}$

National Institute of Chemical Physics and Biophysics, Tallinn, Estonia

M. Kadastik, M. Müntel, M. Murumaa, M. Raidal, L. Rebane, A. Tiko

Department of Physics, University of Helsinki, Helsinki, Finland

P. Eerola, G. Fedi, M. Voutilainen 


\section{Helsinki Institute of Physics, Helsinki, Finland}

J. Härkönen, A. Heikkinen, V. Karimäki, R. Kinnunen, M.J. Kortelainen, T. Lampén, K. Lassila-Perini, S. Lehti, T. Lindén, P. Luukka, T. Mäenpää, T. Peltola, E. Tuominen, J. Tuominiemi, E. Tuovinen, D. Ungaro, L. Wendland

Lappeenranta University of Technology, Lappeenranta, Finland

K. Banzuzi, A. Karjalainen, A. Korpela, T. Tuuva

DSM/IRFU, CEA/Saclay, Gif-sur-Yvette, France

M. Besancon, S. Choudhury, M. Dejardin, D. Denegri, B. Fabbro, J.L. Faure, F. Ferri,

S. Ganjour, A. Givernaud, P. Gras, G. Hamel de Monchenault, P. Jarry, E. Locci, J. Malcles, L. Millischer, A. Nayak, J. Rander, A. Rosowsky, M. Titov

Laboratoire Leprince-Ringuet, Ecole Polytechnique, IN2P3-CNRS, Palaiseau, France

S. Baffioni, F. Beaudette, L. Benhabib, L. Bianchini, M. Bluj ${ }^{13}$, P. Busson, C. Charlot, N. Daci, T. Dahms, M. Dalchenko, L. Dobrzynski, A. Florent, R. Granier de Cassagnac, M. Haguenauer, P. Miné, C. Mironov, I.N. Naranjo, M. Nguyen, C. Ochando, P. Paganini, D. Sabes, R. Salerno, Y. Sirois, C. Veelken, A. Zabi

Institut Pluridisciplinaire Hubert Curien, Université de Strasbourg, Université de Haute Alsace Mulhouse, CNRS/IN2P3, Strasbourg, France

J.-L. Agram ${ }^{14}$, J. Andrea, D. Bloch, D. Bodin, J.-M. Brom, M. Cardaci, E.C. Chabert, C. Collard, E. Conte ${ }^{14}$, F. Drouhin ${ }^{14}$, J.-C. Fontaine ${ }^{14}$, D. Gelé, U. Goerlach, P. Juillot, A.-C. Le Bihan, P. Van Hove

Université de Lyon, Université Claude Bernard Lyon 1, CNRS-IN2P3, Institut de Physique Nucléaire de Lyon, Villeurbanne, France

S. Beauceron, N. Beaupere, O. Bondu, G. Boudoul, S. Brochet, J. Chasserat, R. Chierici ${ }^{2}$, D. Contardo, P. Depasse, H. El Mamouni, J. Fay, S. Gascon, M. Gouzevitch, B. Ille, T. Kurca, M. Lethuillier, L. Mirabito, S. Perries, L. Sgandurra, V. Sordini, Y. Tschudi, P. Verdier, S. Viret

Institute of High Energy Physics and Informatization, Tbilisi State University, Tbilisi, Georgia

Z. Tsamalaidze ${ }^{15}$

RWTH Aachen University, I. Physikalisches Institut, Aachen, Germany

C. Autermann, S. Beranek, B. Calpas, M. Edelhoff, L. Feld, N. Heracleous, O. Hindrichs, R. Jussen, K. Klein, J. Merz, A. Ostapchuk, A. Perieanu, F. Raupach, J. Sammet, S. Schael, D. Sprenger, H. Weber, B. Wittmer, V. Zhukov ${ }^{16}$

RWTH Aachen University, III. Physikalisches Institut A, Aachen, Germany M. Ata, J. Caudron, E. Dietz-Laursonn, D. Duchardt, M. Erdmann, R. Fischer, A. Güth, T. Hebbeker, C. Heidemann, K. Hoepfner, D. Klingebiel, P. Kreuzer, M. Merschmeyer, A. Meyer, M. Olschewski, P. Papacz, H. Pieta, H. Reithler, S.A. Schmitz, L. Sonnenschein, J. Steggemann, D. Teyssier, S. Thüer, M. Weber 
RWTH Aachen University, III. Physikalisches Institut B, Aachen, Germany M. Bontenackels, V. Cherepanov, Y. Erdogan, G. Flügge, H. Geenen, M. Geisler, W. Haj Ahmad, F. Hoehle, B. Kargoll, T. Kress, Y. Kuessel, J. Lingemann², A. Nowack, L. Perchalla, O. Pooth, P. Sauerland, A. Stahl

\section{Deutsches Elektronen-Synchrotron, Hamburg, Germany}

M. Aldaya Martin, J. Behr, W. Behrenhoff, U. Behrens, M. Bergholz ${ }^{17}$, A. Bethani, K. Borras, A. Burgmeier, A. Cakir, L. Calligaris, A. Campbell, E. Castro, F. Costanza, D. Dammann, C. Diez Pardos, T. Dorland, G. Eckerlin, D. Eckstein, G. Flucke, A. Geiser, I. Glushkov, P. Gunnellini, S. Habib, J. Hauk, G. Hellwig, H. Jung, M. Kasemann, P. Katsas, C. Kleinwort, H. Kluge, A. Knutsson, M. Krämer, D. Krücker, E. Kuznetsova, W. Lange, J. Leonard, W. Lohmann ${ }^{17}$, B. Lutz, R. Mankel, I. Marfin, M. Marienfeld, I.-A. Melzer-Pellmann, A.B. Meyer, J. Mnich, A. Mussgiller, S. Naumann-Emme, O. Novgorodova, F. Nowak, J. Olzem, H. Perrey, A. Petrukhin, D. Pitzl, A. Raspereza, P.M. Ribeiro Cipriano, C. Riedl, E. Ron, M. Rosin, J. Salfeld-Nebgen, R. Schmidt ${ }^{17}$, T. Schoerner-Sadenius, N. Sen, A. Spiridonov, M. Stein, R. Walsh, C. Wissing

\section{University of Hamburg, Hamburg, Germany}

V. Blobel, H. Enderle, J. Erfle, U. Gebbert, M. Görner, M. Gosselink, J. Haller, T. Hermanns, R.S. Höing, K. Kaschube, G. Kaussen, H. Kirschenmann, R. Klanner, J. Lange, T. Peiffer, N. Pietsch, D. Rathjens, C. Sander, H. Schettler, P. Schleper, E. Schlieckau, A. Schmidt, M. Schröder, T. Schum, M. Seidel, J. Sibille ${ }^{18}$, V. Sola, H. Stadie, G. Steinbrück, J. Thomsen, L. Vanelderen

\section{Institut für Experimentelle Kernphysik, Karlsruhe, Germany}

C. Barth, J. Berger, C. Böser, T. Chwalek, W. De Boer, A. Descroix, A. Dierlamm, M. Feindt, M. Guthoff ${ }^{2}$, C. Hackstein, F. Hartmann ${ }^{2}$, T. Hauth ${ }^{2}$, M. Heinrich, H. Held, K.H. Hoffmann, U. Husemann, I. Katkov ${ }^{16}$, J.R. Komaragiri, P. Lobelle Pardo, D. Martschei, S. Mueller, Th. Müller, M. Niegel, A. Nürnberg, O. Oberst, A. Oehler, J. Ott, G. Quast, K. Rabbertz, F. Ratnikov, N. Ratnikova, S. Röcker, F.-P. Schilling, G. Schott, H.J. Simonis, F.M. Stober, D. Troendle, R. Ulrich, J. Wagner-Kuhr, S. Wayand, T. Weiler, M. Zeise

\section{Institute of Nuclear Physics "Demokritos", Aghia Paraskevi, Greece}

G. Anagnostou, G. Daskalakis, T. Geralis, S. Kesisoglou, A. Kyriakis, D. Loukas, I. Manolakos, A. Markou, C. Markou, E. Ntomari

University of Athens, Athens, Greece

L. Gouskos, T.J. Mertzimekis, A. Panagiotou, N. Saoulidou

\section{University of Ioánnina, Ioánnina, Greece}

I. Evangelou, C. Foudas, P. Kokkas, N. Manthos, I. Papadopoulos, V. Patras

KFKI Research Institute for Particle and Nuclear Physics, Budapest, Hungary G. Bencze, C. Hajdu, P. Hidas, D. Horvath ${ }^{19}$, F. Sikler, V. Veszpremi, G. Vesztergombi ${ }^{20}$, A.J. Zsigmond 
Institute of Nuclear Research ATOMKI, Debrecen, Hungary

N. Beni, S. Czellar, J. Molnar, J. Palinkas, Z. Szillasi

University of Debrecen, Debrecen, Hungary

J. Karancsi, P. Raics, Z.L. Trocsanyi, B. Ujvari

Panjab University, Chandigarh, India

S.B. Beri, V. Bhatnagar, N. Dhingra, R. Gupta, M. Kaur, M.Z. Mehta, M. Mittal, N. Nishu, L.K. Saini, A. Sharma, J.B. Singh

University of Delhi, Delhi, India

Ashok Kumar, Arun Kumar, S. Ahuja, A. Bhardwaj, B.C. Choudhary, S. Malhotra, M. Naimuddin, K. Ranjan, V. Sharma, R.K. Shivpuri

Saha Institute of Nuclear Physics, Kolkata, India

S. Banerjee, S. Bhattacharya, K. Chatterjee, S. Dutta, B. Gomber, Sa. Jain, Sh. Jain, R. Khurana, S. Mukherjee, D. Roy, S. Sarkar, M. Sharan

Bhabha Atomic Research Centre, Mumbai, India

A. Abdulsalam, D. Dutta, S. Kailas, V. Kumar, A.K. Mohanty², L.M. Pant, P. Shukla

Tata Institute of Fundamental Research - EHEP, Mumbai, India

T. Aziz, S. Ganguly, M. Guchait ${ }^{21}$, A. Gurtu ${ }^{22}$, M. Maity ${ }^{23}$, G. Majumder, K. Mazumdar, G.B. Mohanty, B. Parida, K. Sudhakar, N. Wickramage

Tata Institute of Fundamental Research - HECR, Mumbai, India

S. Banerjee, S. Dugad

Institute for Research in Fundamental Sciences (IPM), Tehran, Iran

H. Arfaei ${ }^{24}$, H. Bakhshiansohi, S.M. Etesami ${ }^{25}$, A. Fahim ${ }^{24}$, M. Hashemi ${ }^{26}$, H. Hesari, A. Jafari, M. Khakzad, M. Mohammadi Najafabadi, S. Paktinat Mehdiabadi, B. Safarzadeh ${ }^{27}$, M. Zeinali

INFN Sezione di Bari ${ }^{a}$, Università di Bari ${ }^{b}$, Politecnico di Bari ${ }^{c}$, Bari, Italy M. Abbrescia ${ }^{a, b}$, L. Barbone ${ }^{a, b}$, C. Calabria ${ }^{a, b, 2}$, S.S. Chhibra ${ }^{a, b}$, A. Colaleo ${ }^{a}$, D. Creanza ${ }^{a, c}$, N. De Filippis ${ }^{a, c, 2}$, M. De Palma ${ }^{a, b}$, L. Fiore $^{a}$, G. Iaselli ${ }^{a, c}$, G. Maggi ${ }^{a, c}$, M. Maggi ${ }^{a}$, B. Marangelli ${ }^{a, b}$, S. My ${ }^{a, c}$, S. $\mathrm{Nuzzo}^{a, b}$, N. Pacifico ${ }^{a}$, A. Pompili ${ }^{a, b}$,

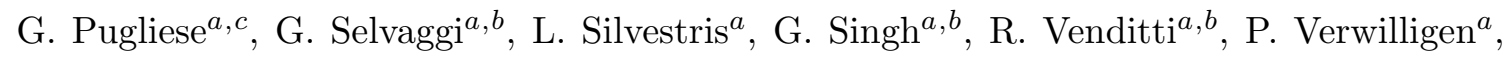
G. Zito ${ }^{a}$

\section{INFN Sezione di Bologna ${ }^{a}$, Università di Bologna ${ }^{b}$, Bologna, Italy}

G. Abbiendi $^{a}$, A.C. Benvenuti ${ }^{a}$, D. Bonacorsi ${ }^{a}, b$, S. Braibant-Giacomelli ${ }^{a, b}$, L. Brigliadori ${ }^{a, b}$, P. Capiluppi ${ }^{a, b}$, A. Castro ${ }^{a, b}$, F.R. Cavallo ${ }^{a}$, M. Cuffiani ${ }^{a, b}$, G.M. Dallavalle ${ }^{a}$, F. Fabbri ${ }^{a}$, A. Fanfani ${ }^{a, b}$, D. Fasanella ${ }^{a, b}$, P. Giacomelli ${ }^{a}$, C. Grandi ${ }^{a}$, L. Guiducci ${ }^{a, b}$, S. Marcellini ${ }^{a}$, G. Masetti ${ }^{a}$, M. Meneghelli ${ }^{a, b, 2}$, A. Montanari ${ }^{a}$,

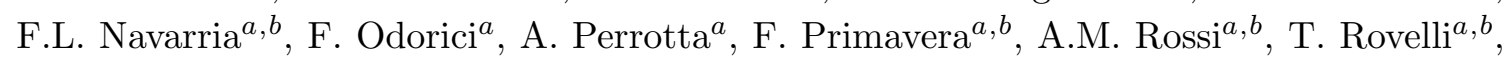
G.P. Siroli ${ }^{a, b}$, N. Tosi, R. Travaglini ${ }^{a, b}$ 
INFN Sezione di Catania ${ }^{a}$, Università di Catania ${ }^{b}$, Catania, Italy

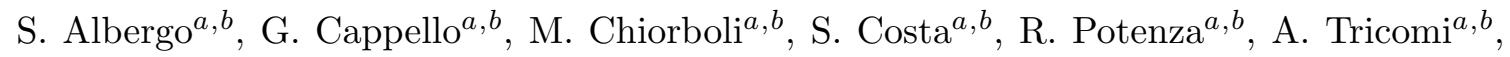
C. Tuve ${ }^{a, b}$

INFN Sezione di Firenze ${ }^{a}$, Università di Firenze ${ }^{b}$, Firenze, Italy

G. Barbagli ${ }^{a}$, V. Ciulli ${ }^{a, b}$, C. Civinini ${ }^{a}$, R. D’Alessandro ${ }^{a, b}$, E. Focardi ${ }^{a}, b$, S. Frosali ${ }^{a, b}$,

E. Gallo ${ }^{a}$, S. Gonzi ${ }^{a}, b$, M. Meschini ${ }^{a}$, S. Paoletti ${ }^{a}$, G. Sguazzoni $^{a}$, A. Tropiano ${ }^{a, b}$

INFN Laboratori Nazionali di Frascati, Frascati, Italy

L. Benussi, S. Bianco, S. Colafranceschi ${ }^{28}$, F. Fabbri, D. Piccolo

INFN Sezione di Genova ${ }^{a}$, Università di Genova ${ }^{b}$, Genova, Italy

P. Fabbricatore ${ }^{a}$, R. Musenich ${ }^{a}$, S. Tosi ${ }^{a, b}$

INFN Sezione di Milano-Bicocca ${ }^{a}$, Università di Milano-Bicocca ${ }^{b}$, Milano, Italy

A. Benaglia ${ }^{a}$, F. De Guio ${ }^{a, b}$, L. Di Matteo ${ }^{a, b, 2}$, S. Fiorendi ${ }^{a, b}$, S. Gennai ${ }^{a, 2}$, A. Ghezzi $^{a, b}$, S. Malvezzi ${ }^{a}$, R.A. Manzoni ${ }^{a, b}$, A. Martelli ${ }^{a, b}$, A. Massironi ${ }^{a, b}$, D. Menasce ${ }^{a}$, L. Moroni ${ }^{a}$, M. Paganoni ${ }^{a, b}$, D. Pedrini ${ }^{a}$, S. Ragazzi ${ }^{a, b}$, N. Redaelli ${ }^{a}$, S. Sala ${ }^{a}$, T. Tabarelli de Fatis ${ }^{a, b}$

INFN Sezione di Napoli ${ }^{a}$, Università di Napoli "Federico II" $b$, Napoli, Italy

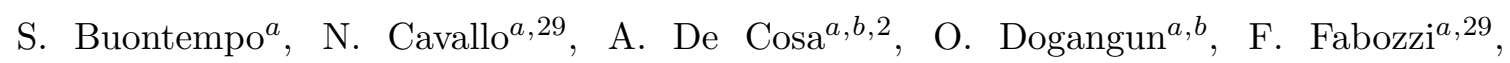
A.O.M. Iorio ${ }^{a, b}$, L. Lista $^{a}$, S. Meola ${ }^{a, 30}$, M. Merola ${ }^{a}$, P. Paolucci ${ }^{a, 2}$

INFN Sezione di Padova ${ }^{a}$, Università di Padova ${ }^{b}$, Università di Trento (Trento) ${ }^{c}$, Padova, Italy

P. Azzi ${ }^{a}$, N. Bacchetta ${ }^{a, 2}$, P. Bellan ${ }^{a, b}$, D. Bisello ${ }^{a, b}$ A. Branca ${ }^{a, b, 2}$, R. Carlin $^{a, b}$, P. Checchia ${ }^{a}$, T. Dorigo ${ }^{a}$, U. Dosselli ${ }^{a}$, F. Gasparini ${ }^{a, b}$, U. Gasparini ${ }^{a, b}$, A. Gozzelino ${ }^{a}$, K. Kanishchev ${ }^{a, c}$, S. Lacaprara ${ }^{a}$, I. Lazzizzera ${ }^{a, c}$, M. Margoni $^{a, b}$, A.T. Meneguzzo ${ }^{a, b}$, M. Nespolo ${ }^{a, 2}$, J. Pazzini ${ }^{a, b}$, P. Ronchese ${ }^{a, b}$, F. Simonetto ${ }^{a, b}$, E. Torassa ${ }^{a}$, S. Vanini ${ }^{a, b}$, P. Zotto ${ }^{a, b}$, G. Zumerle ${ }^{a, b}$

INFN Sezione di Pavia ${ }^{a}$, Università di Pavia ${ }^{b}$, Pavia, Italy

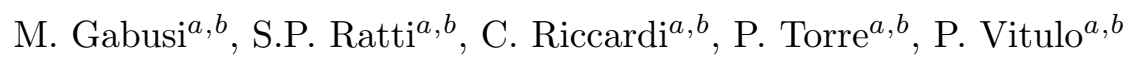

INFN Sezione di Perugia ${ }^{a}$, Università di Perugia ${ }^{b}$, Perugia, Italy

M. Biasini ${ }^{a}, b$, G.M. Bilei ${ }^{a}$, L. Fanò ${ }^{a, b}$, P. Lariccia ${ }^{a, b}$, G. Mantovani ${ }^{a}, b$, M. Menichelli ${ }^{a}$,

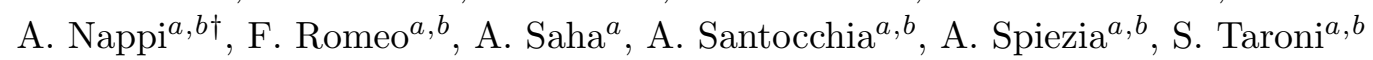

INFN Sezione di Pisa ${ }^{a}$, Università di Pisa ${ }^{b}$, Scuola Normale Superiore di Pisa ${ }^{c}$, Pisa, Italy

P. Azzurri ${ }^{a, c}$, G. Bagliesi ${ }^{a}$, J. Bernardini ${ }^{a}$, T. Boccali ${ }^{a}$, G. Broccolo ${ }^{a, c}$, R. Castaldi $^{a}$, R.T. D'Agnolo ${ }^{a, c, 2}$, R. Dell'Orso ${ }^{a}$, F. Fiori ${ }^{a, b, 2}$, L. Foà ${ }^{a, c}$, A. Giassi ${ }^{a}$, A. Kraan ${ }^{a}$, F. $\operatorname{Ligabue}^{a, c}$, T. $\operatorname{Lomtadze}^{a}$, L. Martini ${ }^{a, 31}$, A. Messineo ${ }^{a, b}$, F. Palla ${ }^{a}$, A. Rizzi ${ }^{a, b}$,

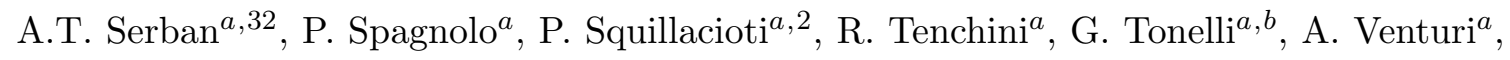
P.G. Verdini ${ }^{a}$ 
INFN Sezione di Roma ${ }^{a}$, Università di Roma ${ }^{b}$, Roma, Italy

L. Barone ${ }^{a, b}$, F. Cavallari ${ }^{a}$, D. Del Re $\mathrm{Re}^{a, b}$, M. Diemoz ${ }^{a}$, C. Fanellia ${ }^{a, b}$, M. Grassi ${ }^{a, b, 2}$, E. Longo $^{a, b}$, P. Meridiani ${ }^{a, 2}$, F. Micheli ${ }^{a, b}$, S. Nourbakhsh ${ }^{a, b}$, G. Organtini $^{a, b}$, R. Paramatti ${ }^{a}$, S. Rahatlou ${ }^{a, b}$, L. Soffi ${ }^{a, b}$

INFN Sezione di Torino ${ }^{a}$, Università di Torino ${ }^{b}$, Università del Piemonte Orientale (Novara) ${ }^{c}$, Torino, Italy

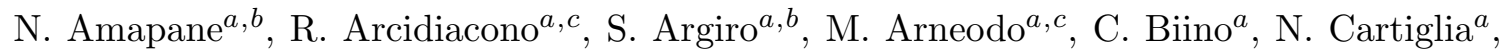

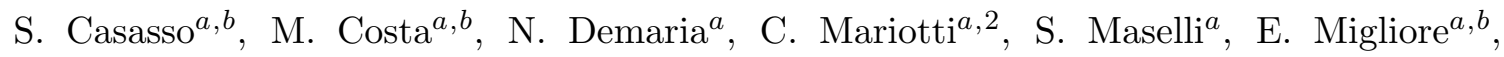
V. Monaco $^{a, b}$, M. Musich ${ }^{a, 2}$, M.M. Obertino ${ }^{a, c}$, N. Pastrone ${ }^{a}$, M. Pelliccioni ${ }^{a}$, A. Potenza ${ }^{a, b}$, A. Romero ${ }^{a, b}$, M. Ruspa ${ }^{a, c}$, R. Sacchi ${ }^{a, b}$, A. Solano ${ }^{a, b}$, A. Staiano ${ }^{a}$

INFN Sezione di Trieste ${ }^{a}$, Università di Trieste ${ }^{b}$, Trieste, Italy

S. Belforte ${ }^{a}$, V. Candelise ${ }^{a, b}$, M. Casarsa ${ }^{a}$, F. Cossutti ${ }^{a}$, G. Della Ricca ${ }^{a, b}$, B. Gobbo ${ }^{a}$, M. Marone ${ }^{a, b, 2}$, D. Montanino ${ }^{a, b, 2}$, A. Penzo ${ }^{a}$, A. Schizzi ${ }^{a, b}$

Kangwon National University, Chunchon, Korea

T.Y. Kim, S.K. Nam

Kyungpook National University, Daegu, Korea

S. Chang, D.H. Kim, G.N. Kim, D.J. Kong, H. Park, D.C. Son, T. Son

Chonnam National University, Institute for Universe and Elementary Particles, Kwangju, Korea

J.Y. Kim, Zero J. Kim, S. Song

Korea University, Seoul, Korea

S. Choi, D. Gyun, B. Hong, M. Jo, H. Kim, T.J. Kim, K.S. Lee, D.H. Moon, S.K. Park, Y. Roh

University of Seoul, Seoul, Korea

M. Choi, J.H. Kim, C. Park, I.C. Park, S. Park, G. Ryu

Sungkyunkwan University, Suwon, Korea

Y. Choi, Y.K. Choi, J. Goh, M.S. Kim, E. Kwon, B. Lee, J. Lee, S. Lee, H. Seo, I. Yu

Vilnius University, Vilnius, Lithuania

M.J. Bilinskas, I. Grigelionis, M. Janulis, A. Juodagalvis

Centro de Investigacion y de Estudios Avanzados del IPN, Mexico City, Mexico

H. Castilla-Valdez, E. De La Cruz-Burelo, I. Heredia-de La Cruz, R. Lopez-Fernandez, J. Martínez-Ortega, A. Sánchez-Hernández, L.M. Villasenor-Cendejas

Universidad Iberoamericana, Mexico City, Mexico

S. Carrillo Moreno, F. Vazquez Valencia

Benemerita Universidad Autonoma de Puebla, Puebla, Mexico H.A. Salazar Ibarguen 
Universidad Autónoma de San Luis Potosí, San Luis Potosí, Mexico

E. Casimiro Linares, A. Morelos Pineda, M.A. Reyes-Santos

University of Auckland, Auckland, New Zealand

D. Krofcheck

University of Canterbury, Christchurch, New Zealand

A.J. Bell, P.H. Butler, R. Doesburg, S. Reucroft, H. Silverwood

National Centre for Physics, Quaid-I-Azam University, Islamabad, Pakistan

M. Ahmad, M.I. Asghar, J. Butt, H.R. Hoorani, S. Khalid, W.A. Khan, T. Khurshid, S. Qazi, M.A. Shah, M. Shoaib

National Centre for Nuclear Research, Swierk, Poland

H. Bialkowska, B. Boimska, T. Frueboes, M. Górski, M. Kazana, K. Nawrocki, K. Romanowska-Rybinska, M. Szleper, G. Wrochna, P. Zalewski

Institute of Experimental Physics, Faculty of Physics, University of Warsaw, Warsaw, Poland

G. Brona, K. Bunkowski, M. Cwiok, W. Dominik, K. Doroba, A. Kalinowski, M. Konecki, J. Krolikowski, M. Misiura

Laboratório de Instrumentação e Física Experimental de Partículas, Lisboa, Portugal

N. Almeida, P. Bargassa, A. David, P. Faccioli, P.G. Ferreira Parracho, M. Gallinaro, J. Seixas, J. Varela, P. Vischia

Joint Institute for Nuclear Research, Dubna, Russia

I. Belotelov, P. Bunin, M. Gavrilenko, I. Golutvin, I. Gorbunov, A. Kamenev, V. Karjavin, G. Kozlov, A. Lanev, A. Malakhov, P. Moisenz, V. Palichik, V. Perelygin, S. Shmatov, V. Smirnov, A. Volodko, A. Zarubin

Petersburg Nuclear Physics Institute, Gatchina (St. Petersburg), Russia

S. Evstyukhin, V. Golovtsov, Y. Ivanov, V. Kim, P. Levchenko, V. Murzin, V. Oreshkin, I. Smirnov, V. Sulimov, L. Uvarov, S. Vavilov, A. Vorobyev, An. Vorobyev

Institute for Nuclear Research, Moscow, Russia

Yu. Andreev, A. Dermenev, S. Gninenko, N. Golubev, M. Kirsanov, N. Krasnikov, V. Matveev, A. Pashenkov, D. Tlisov, A. Toropin

Institute for Theoretical and Experimental Physics, Moscow, Russia

V. Epshteyn, M. Erofeeva, V. Gavrilov, M. Kossov, N. Lychkovskaya, V. Popov, G. Safronov, S. Semenov, I. Shreyber, V. Stolin, E. Vlasov, A. Zhokin

Moscow State University, Moscow, Russia

A. Belyaev, E. Boos, M. Dubinin ${ }^{5}$, L. Dudko, A. Ershov, A. Gribushin, V. Klyukhin, O. Kodolova, I. Lokhtin, A. Markina, S. Obraztsov, M. Perfilov, S. Petrushanko, A. Popov, L. Sarycheva ${ }^{\dagger}$, V. Savrin, A. Snigirev 
P.N. Lebedev Physical Institute, Moscow, Russia

V. Andreev, M. Azarkin, I. Dremin, M. Kirakosyan, A. Leonidov, G. Mesyats, S.V. Rusakov, A. Vinogradov

State Research Center of Russian Federation, Institute for High Energy Physics, Protvino, Russia

I. Azhgirey, I. Bayshev, S. Bitioukov, V. Grishin ${ }^{2}$, V. Kachanov, D. Konstantinov,

V. Krychkine, V. Petrov, R. Ryutin, A. Sobol, L. Tourtchanovitch, S. Troshin, N. Tyurin,

A. Uzunian, A. Volkov

University of Belgrade, Faculty of Physics and Vinca Institute of Nuclear Sciences, Belgrade, Serbia

P. Adzic ${ }^{33}$, M. Djordjevic, M. Ekmedzic, D. Krpic ${ }^{33}$, J. Milosevic

Centro de Investigaciones Energéticas Medioambientales y Tecnológicas (CIEMAT), Madrid, Spain

M. Aguilar-Benitez, J. Alcaraz Maestre, P. Arce, C. Battilana, E. Calvo, M. Cerrada,

M. Chamizo Llatas, N. Colino, B. De La Cruz, A. Delgado Peris, D. Domínguez Vázquez,

C. Fernandez Bedoya, J.P. Fernández Ramos, A. Ferrando, J. Flix, M.C. Fouz, P. GarciaAbia, O. Gonzalez Lopez, S. Goy Lopez, J.M. Hernandez, M.I. Josa, G. Merino, J. Puerta Pelayo, A. Quintario Olmeda, I. Redondo, L. Romero, J. Santaolalla, M.S. Soares, C. Willmott

Universidad Autónoma de Madrid, Madrid, Spain

C. Albajar, G. Codispoti, J.F. de Trocóniz

Universidad de Oviedo, Oviedo, Spain

H. Brun, J. Cuevas, J. Fernandez Menendez, S. Folgueras, I. Gonzalez Caballero, L. Lloret Iglesias, J. Piedra Gomez

Instituto de Física de Cantabria (IFCA), CSIC-Universidad de Cantabria, Santander, Spain

J.A. Brochero Cifuentes, I.J. Cabrillo, A. Calderon, S.H. Chuang, J. Duarte Campderros, M. Felcini ${ }^{34}$, M. Fernandez, G. Gomez, J. Gonzalez Sanchez, A. Graziano, C. Jorda, A. Lopez Virto, J. Marco, R. Marco, C. Martinez Rivero, F. Matorras, F.J. Munoz Sanchez, T. Rodrigo, A.Y. Rodríguez-Marrero, A. Ruiz-Jimeno, L. Scodellaro, I. Vila, R. Vilar Cortabitarte

\section{CERN, European Organization for Nuclear Research, Geneva, Switzerland}

D. Abbaneo, E. Auffray, G. Auzinger, M. Bachtis, P. Baillon, A.H. Ball, D. Barney, J.F. Benitez, C. Bernet ${ }^{6}$, G. Bianchi, P. Bloch, A. Bocci, A. Bonato, C. Botta, H. Breuker, T. Camporesi, G. Cerminara, T. Christiansen, J.A. Coarasa Perez, D. D'Enterria, A. Dabrowski, A. De Roeck, S. Di Guida, M. Dobson, N. Dupont-Sagorin, A. ElliottPeisert, B. Frisch, W. Funk, G. Georgiou, M. Giffels, D. Gigi, K. Gill, D. Giordano, M. Girone, M. Giunta, F. Glege, R. Gomez-Reino Garrido, P. Govoni, S. Gowdy, R. Guida,

S. Gundacker, J. Hammer, M. Hansen, P. Harris, C. Hartl, J. Harvey, B. Hegner, A. Hinzmann, V. Innocente, P. Janot, K. Kaadze, E. Karavakis, K. Kousouris, P. Lecoq, 
Y.-J. Lee, P. Lenzi, C. Lourenço, N. Magini, T. Mäki, M. Malberti, L. Malgeri, M. Mannelli, L. Masetti, F. Meijers, S. Mersi, E. Meschi, R. Moser, M. Mulders, P. Musella, E. Nesvold, L. Orsini, E. Palencia Cortezon, E. Perez, L. Perrozzi, A. Petrilli, A. Pfeiffer, M. Pierini, M. Pimiä, D. Piparo, G. Polese, L. Quertenmont, A. Racz, W. Reece, J. Rodrigues Antunes, G. Rolandi ${ }^{35}$, C. Rovelli ${ }^{36}$, M. Rovere, H. Sakulin, F. Santanastasio, C. Schäfer, C. Schwick, I. Segoni, S. Sekmen, A. Sharma, P. Siegrist, P. Silva, M. Simon, P. Sphicas ${ }^{37}$, D. Spiga, A. Tsirou, G.I. Veres ${ }^{20}$, J.R. Vlimant, H.K. Wöhri, S.D. Worm ${ }^{38}$, W.D. Zeuner

\section{Paul Scherrer Institut, Villigen, Switzerland}

W. Bertl, K. Deiters, W. Erdmann, K. Gabathuler, R. Horisberger, Q. Ingram, H.C. Kaestli, S. König, D. Kotlinski, U. Langenegger, F. Meier, D. Renker, T. Rohe

Institute for Particle Physics, ETH Zurich, Zurich, Switzerland

L. Bäni, P. Bortignon, M.A. Buchmann, B. Casal, N. Chanon, A. Deisher, G. Dissertori, M. Dittmar, M. Donegà, M. Dünser, P. Eller, J. Eugster, K. Freudenreich, C. Grab, D. Hits, P. Lecomte, W. Lustermann, A.C. Marini, P. Martinez Ruiz del Arbol, N. Mohr, F. Moortgat, C. Nägeli ${ }^{39}$, P. Nef, F. Nessi-Tedaldi, F. Pandolfi, L. Pape, F. Pauss, M. Peruzzi, F.J. Ronga, M. Rossini, L. Sala, A.K. Sanchez, A. Starodumov ${ }^{40}$, B. Stieger, M. Takahashi, L. Tauscher ${ }^{\dagger}$, A. Thea, K. Theofilatos, D. Treille, C. Urscheler, R. Wallny, H.A. Weber, L. Wehrli

\section{Universität Zürich, Zurich, Switzerland}

C. Amsler ${ }^{41}$, V. Chiochia, S. De Visscher, C. Favaro, M. Ivova Rikova, B. Kilminster, B. Millan Mejias, P. Otiougova, P. Robmann, H. Snoek, S. Tupputi, M. Verzetti

\section{National Central University, Chung-Li, Taiwan}

Y.H. Chang, K.H. Chen, C. Ferro, C.M. Kuo, S.W. Li, W. Lin, Y.J. Lu, A.P. Singh, R. Volpe, S.S. Yu

\section{National Taiwan University (NTU), Taipei, Taiwan}

P. Bartalini, P. Chang, Y.H. Chang, Y.W. Chang, Y. Chao, K.F. Chen, C. Dietz, U. Grundler, W.-S. Hou, Y. Hsiung, K.Y. Kao, Y.J. Lei, R.-S. Lu, D. Majumder, E. Petrakou, X. Shi, J.G. Shiu, Y.M. Tzeng, X. Wan, M. Wang

\section{Chulalongkorn University, Bangkok, Thailand}

B. Asavapibhop, N. Srimanobhas, N. Suwonjandee

\section{Cukurova University, Adana, Turkey}

A. Adiguzel, M.N. Bakirci ${ }^{42}$, S. $\mathrm{Cerci}^{43}$, C. Dozen, I. Dumanoglu, E. Eskut, S. Girgis, G. Gokbulut, E. Gurpinar, I. Hos, E.E. Kangal, T. Karaman, G. Karapinar ${ }^{44}$, A. Kayis Topaksu, G. Onengut, K. Ozdemir, S. Ozturk ${ }^{45}$, A. Polatoz, K. Sogut ${ }^{46}$, D. Sunar Cerci ${ }^{43}$, B. Tali ${ }^{43}$, H. Topakli ${ }^{42}$, L.N. Vergili, M. Vergili

Middle East Technical University, Physics Department, Ankara, Turkey I.V. Akin, T. Aliev, B. Bilin, S. Bilmis, M. Deniz, H. Gamsizkan, A.M. Guler, K. Ocalan, A. Ozpineci, M. Serin, R. Sever, U.E. Surat, M. Yalvac, E. Yildirim, M. Zeyrek 
Bogazici University, Istanbul, Turkey

E. Gülmez, B. Isildak ${ }^{47}$, M. Kaya ${ }^{48}$, O. Kaya ${ }^{48}$, S. Ozkorucuklu ${ }^{49}$, N. Sonmez ${ }^{50}$

Istanbul Technical University, Istanbul, Turkey

H. Bahtiyar, E. Barlas, K. Cankocak, Y.O. Günaydin, F.I. Vardarlı, M. Yücel

National Scientific Center, Kharkov Institute of Physics and Technology, Kharkov, Ukraine

L. Levchuk

University of Bristol, Bristol, United Kingdom

J.J. Brooke, E. Clement, D. Cussans, H. Flacher, R. Frazier, J. Goldstein, M. Grimes, G.P. Heath, H.F. Heath, L. Kreczko, S. Metson, D.M. Newbold ${ }^{38}$, K. Nirunpong, A. Poll, S. Senkin, V.J. Smith, T. Williams

Rutherford Appleton Laboratory, Didcot, United Kingdom

L. Basso ${ }^{51}$, K.W. Bell, A. Belyaev ${ }^{51}$, C. Brew, R.M. Brown, D.J.A. Cockerill, J.A. Coughlan, K. Harder, S. Harper, J. Jackson, B.W. Kennedy, E. Olaiya, D. Petyt, B.C. RadburnSmith, C.H. Shepherd-Themistocleous, I.R. Tomalin, W.J. Womersley

\section{Imperial College, London, United Kingdom}

R. Bainbridge, G. Ball, R. Beuselinck, O. Buchmuller, D. Colling, N. Cripps, M. Cutajar, P. Dauncey, G. Davies, M. Della Negra, W. Ferguson, J. Fulcher, D. Futyan, A. Gilbert,

A. Guneratne Bryer, G. Hall, Z. Hatherell, J. Hays, G. Iles, M. Jarvis, G. Karapostoli, L. Lyons, A.-M. Magnan, J. Marrouche, B. Mathias, R. Nandi, J. Nash, A. Nikitenko ${ }^{40}$, J. Pela, M. Pesaresi, K. Petridis, M. Pioppi ${ }^{52}$, D.M. Raymond, S. Rogerson, A. Rose, M.J. Ryan, C. Seez, P. Sharp ${ }^{\dagger}$, A. Sparrow, M. Stoye, A. Tapper, M. Vazquez Acosta, T. Virdee, S. Wakefield, N. Wardle, T. Whyntie

\section{Brunel University, Uxbridge, United Kingdom}

M. Chadwick, J.E. Cole, P.R. Hobson, A. Khan, P. Kyberd, D. Leggat, D. Leslie, W. Martin, I.D. Reid, P. Symonds, L. Teodorescu, M. Turner

Baylor University, Waco, U.S.A.

K. Hatakeyama, H. Liu, T. Scarborough

The University of Alabama, Tuscaloosa, U.S.A.

O. Charaf, C. Henderson, P. Rumerio

Boston University, Boston, U.S.A.

A. Avetisyan, T. Bose, C. Fantasia, A. Heister, J. St. John, P. Lawson, D. Lazic, J. Rohlf, D. Sperka, L. Sulak

Brown University, Providence, U.S.A.

J. Alimena, S. Bhattacharya, G. Christopher, D. Cutts, Z. Demiragli, A. Ferapontov, A. Garabedian, U. Heintz, S. Jabeen, G. Kukartsev, E. Laird, G. Landsberg, M. Luk, M. Narain, D. Nguyen, M. Segala, T. Sinthuprasith, T. Speer 
University of California, Davis, Davis, U.S.A.

R. Breedon, G. Breto, M. Calderon De La Barca Sanchez, S. Chauhan, M. Chertok, J. Conway, R. Conway, P.T. Cox, J. Dolen, R. Erbacher, M. Gardner, R. Houtz, W. Ko, A. Kopecky, R. Lander, O. Mall, T. Miceli, D. Pellett, F. Ricci-Tam, B. Rutherford, M. Searle, J. Smith, M. Squires, M. Tripathi, R. Vasquez Sierra, R. Yohay

University of California, Los Angeles, Los Angeles, U.S.A.

V. Andreev, D. Cline, R. Cousins, J. Duris, S. Erhan, P. Everaerts, C. Farrell, J. Hauser, M. Ignatenko, C. Jarvis, G. Rakness, P. Schlein ${ }^{\dagger}$, P. Traczyk, V. Valuev, M. Weber

University of California, Riverside, Riverside, U.S.A.

J. Babb, R. Clare, M.E. Dinardo, J. Ellison, J.W. Gary, F. Giordano, G. Hanson, H. Liu, O.R. Long, A. Luthra, H. Nguyen, S. Paramesvaran, J. Sturdy, S. Sumowidagdo, R. Wilken, S. Wimpenny

University of California, San Diego, La Jolla, U.S.A.

W. Andrews, J.G. Branson, G.B. Cerati, S. Cittolin, D. Evans, A. Holzner, R. Kelley, M. Lebourgeois, J. Letts, I. Macneill, B. Mangano, S. Padhi, C. Palmer, G. Petrucciani, M. Pieri, M. Sani, V. Sharma, S. Simon, E. Sudano, M. Tadel, Y. Tu, A. Vartak, S. Wasserbaech ${ }^{53}$, F. Würthwein, A. Yagil, J. Yoo

University of California, Santa Barbara, Santa Barbara, U.S.A.

D. Barge, R. Bellan, C. Campagnari, M. D’Alfonso, T. Danielson, K. Flowers, P. Geffert, C. George, F. Golf, J. Incandela, C. Justus, P. Kalavase, D. Kovalskyi, V. Krutelyov, S. Lowette, R. Magaña Villalba, N. Mccoll, V. Pavlunin, J. Ribnik, J. Richman, R. Rossin, D. Stuart, W. To, C. West

California Institute of Technology, Pasadena, U.S.A.

A. Apresyan, A. Bornheim, Y. Chen, E. Di Marco, J. Duarte, M. Gataullin, Y. Ma, A. Mott, H.B. Newman, C. Rogan, M. Spiropulu, V. Timciuc, J. Veverka, R. Wilkinson, S. Xie, Y. Yang, R.Y. Zhu

\section{Carnegie Mellon University, Pittsburgh, U.S.A.}

V. Azzolini, A. Calamba, R. Carroll, T. Ferguson, Y. Iiyama, D.W. Jang, Y.F. Liu, M. Paulini, H. Vogel, I. Vorobiev

University of Colorado at Boulder, Boulder, U.S.A.

J.P. Cumalat, B.R. Drell, W.T. Ford, A. Gaz, E. Luiggi Lopez, J.G. Smith, K. Stenson, K.A. Ulmer, S.R. Wagner

Cornell University, Ithaca, U.S.A.

J. Alexander, A. Chatterjee, N. Eggert, L.K. Gibbons, B. Heltsley, W. Hopkins, A. Khukhunaishvili, B. Kreis, N. Mirman, G. Nicolas Kaufman, J.R. Patterson, A. Ryd, E. Salvati, W. Sun, W.D. Teo, J. Thom, J. Thompson, J. Tucker, J. Vaughan, Y. Weng, L. Winstrom, P. Wittich

Fairfield University, Fairfield, U.S.A.

D. Winn 
Fermi National Accelerator Laboratory, Batavia, U.S.A.

S. Abdullin, M. Albrow, J. Anderson, L.A.T. Bauerdick, A. Beretvas, J. Berryhill, P.C. Bhat, K. Burkett, J.N. Butler, V. Chetluru, H.W.K. Cheung, F. Chlebana, V.D. Elvira, I. Fisk, J. Freeman, Y. Gao, D. Green, O. Gutsche, J. Hanlon, R.M. Harris, J. Hirschauer, B. Hooberman, S. Jindariani, M. Johnson, U. Joshi, B. Klima, S. Kunori, S. Kwan, C. Leonidopoulos ${ }^{54}$, J. Linacre, D. Lincoln, R. Lipton, J. Lykken, K. Maeshima, J.M. Marraffino, S. Maruyama, D. Mason, P. McBride, K. Mishra, S. Mrenna, Y. Musienko ${ }^{55}$, C. Newman-Holmes, V. O'Dell, O. Prokofyev, E. SextonKennedy, S. Sharma, W.J. Spalding, L. Spiegel, L. Taylor, S. Tkaczyk, N.V. Tran, L. Uplegger, E.W. Vaandering, R. Vidal, J. Whitmore, W. Wu, F. Yang, J.C. Yun

University of Florida, Gainesville, U.S.A.

D. Acosta, P. Avery, D. Bourilkov, M. Chen, T. Cheng, S. Das, M. De Gruttola, G.P. Di Giovanni, D. Dobur, A. Drozdetskiy, R.D. Field, M. Fisher, Y. Fu, I.K. Furic, J. Gartner, J. Hugon, B. Kim, J. Konigsberg, A. Korytov, A. Kropivnitskaya, T. Kypreos, J.F. Low, K. Matchev, P. Milenovic ${ }^{56}$, G. Mitselmakher, L. Muniz, M. Park, R. Remington, A. Rinkevicius, P. Sellers, N. Skhirtladze, M. Snowball, J. Yelton, M. Zakaria

Florida International University, Miami, U.S.A.

V. Gaultney, S. Hewamanage, L.M. Lebolo, S. Linn, P. Markowitz, G. Martinez, J.L. Rodriguez

Florida State University, Tallahassee, U.S.A.

T. Adams, A. Askew, J. Bochenek, J. Chen, B. Diamond, S.V. Gleyzer, J. Haas, S. Hagopian, V. Hagopian, M. Jenkins, K.F. Johnson, H. Prosper, V. Veeraraghavan, M. Weinberg

Florida Institute of Technology, Melbourne, U.S.A.

M.M. Baarmand, B. Dorney, M. Hohlmann, H. Kalakhety, I. Vodopiyanov, F. Yumiceva

University of Illinois at Chicago (UIC), Chicago, U.S.A.

M.R. Adams, I.M. Anghel, L. Apanasevich, Y. Bai, V.E. Bazterra, R.R. Betts, I. Bucinskaite, J. Callner, R. Cavanaugh, O. Evdokimov, L. Gauthier, C.E. Gerber, D.J. Hofman, S. Khalatyan, F. Lacroix, C. O'Brien, C. Silkworth, D. Strom, P. Turner, N. Varelas

The University of Iowa, Iowa City, U.S.A.

U. Akgun, E.A. Albayrak, B. Bilki ${ }^{57}$, W. Clarida, F. Duru, S. Griffiths, J.-P. Merlo, H. Mermerkaya ${ }^{58}$, A. Mestvirishvili, A. Moeller, J. Nachtman, C.R. Newsom, E. Norbeck, Y. Onel, F. Ozok ${ }^{59}$, S. Sen, P. Tan, E. Tiras, J. Wetzel, T. Yetkin, K. Yi

Johns Hopkins University, Baltimore, U.S.A.

B.A. Barnett, B. Blumenfeld, S. Bolognesi, D. Fehling, G. Giurgiu, A.V. Gritsan, Z.J. Guo, G. Hu, P. Maksimovic, M. Swartz, A. Whitbeck

The University of Kansas, Lawrence, U.S.A.

P. Baringer, A. Bean, G. Benelli, R.P. Kenny Iii, M. Murray, D. Noonan, S. Sanders, R. Stringer, G. Tinti, J.S. Wood 
Kansas State University, Manhattan, U.S.A.

A.F. Barfuss, T. Bolton, I. Chakaberia, A. Ivanov, S. Khalil, M. Makouski, Y. Maravin, S. Shrestha, I. Svintradze

Lawrence Livermore National Laboratory, Livermore, U.S.A.

J. Gronberg, D. Lange, F. Rebassoo, D. Wright

University of Maryland, College Park, U.S.A.

A. Baden, B. Calvert, S.C. Eno, J.A. Gomez, N.J. Hadley, R.G. Kellogg, M. Kirn,

T. Kolberg, Y. Lu, M. Marionneau, A.C. Mignerey, K. Pedro, A. Peterman, A. Skuja,

J. Temple, M.B. Tonjes, S.C. Tonwar

Massachusetts Institute of Technology, Cambridge, U.S.A.

A. Apyan, G. Bauer, J. Bendavid, W. Busza, E. Butz, I.A. Cali, M. Chan, V. Dutta, G. Gomez Ceballos, M. Goncharov, Y. Kim, M. Klute, K. Krajczar ${ }^{60}$, A. Levin, P.D. Luckey, T. Ma, S. Nahn, C. Paus, D. Ralph, C. Roland, G. Roland, M. Rudolph, G.S.F. Stephans, F. Stöckli, K. Sumorok, K. Sung, D. Velicanu, E.A. Wenger, R. Wolf, B. Wyslouch, M. Yang, Y. Yilmaz, A.S. Yoon, M. Zanetti, V. Zhukova

University of Minnesota, Minneapolis, U.S.A.

S.I. Cooper, B. Dahmes, A. De Benedetti, G. Franzoni, A. Gude, S.C. Kao, K. Klapoetke, Y. Kubota, J. Mans, N. Pastika, R. Rusack, M. Sasseville, A. Singovsky, N. Tambe, J. Turkewitz

University of Mississippi, Oxford, U.S.A.

L.M. Cremaldi, R. Kroeger, L. Perera, R. Rahmat, D.A. Sanders

University of Nebraska-Lincoln, Lincoln, U.S.A.

E. Avdeeva, K. Bloom, S. Bose, D.R. Claes, A. Dominguez, M. Eads, J. Keller, I. Kravchenko, J. Lazo-Flores, S. Malik, G.R. Snow

State University of New York at Buffalo, Buffalo, U.S.A.

A. Godshalk, I. Iashvili, S. Jain, A. Kharchilava, A. Kumar, S. Rappoccio

Northeastern University, Boston, U.S.A.

G. Alverson, E. Barberis, D. Baumgartel, M. Chasco, J. Haley, D. Nash, T. Orimoto, D. Trocino, D. Wood, J. Zhang

Northwestern University, Evanston, U.S.A.

A. Anastassov, K.A. Hahn, A. Kubik, L. Lusito, N. Mucia, N. Odell, R.A. Ofierzynski, B. Pollack, A. Pozdnyakov, M. Schmitt, S. Stoynev, M. Velasco, S. Won

University of Notre Dame, Notre Dame, U.S.A.

L. Antonelli, D. Berry, A. Brinkerhoff, K.M. Chan, M. Hildreth, C. Jessop, D.J. Karmgard, J. Kolb, K. Lannon, W. Luo, S. Lynch, N. Marinelli, D.M. Morse, T. Pearson, M. Planer, R. Ruchti, J. Slaunwhite, N. Valls, M. Wayne, M. Wolf 
The Ohio State University, Columbus, U.S.A.

B. Bylsma, L.S. Durkin, C. Hill, R. Hughes, K. Kotov, T.Y. Ling, D. Puigh, M. Rodenburg, C. Vuosalo, G. Williams, B.L. Winer

Princeton University, Princeton, U.S.A.

E. Berry, P. Elmer, V. Halyo, P. Hebda, J. Hegeman, A. Hunt, P. Jindal, S.A. Koay, D. Lopes Pegna, P. Lujan, D. Marlow, T. Medvedeva, M. Mooney, J. Olsen, P. Piroué, X. Quan, A. Raval, H. Saka, D. Stickland, C. Tully, J.S. Werner, S.C. Zenz, A. Zuranski

University of Puerto Rico, Mayaguez, U.S.A.

E. Brownson, A. Lopez, H. Mendez, J.E. Ramirez Vargas

Purdue University, West Lafayette, U.S.A.

E. Alagoz, V.E. Barnes, D. Benedetti, G. Bolla, D. Bortoletto, M. De Mattia, A. Everett, Z. Hu, M. Jones, O. Koybasi, M. Kress, A.T. Laasanen, N. Leonardo, V. Maroussov, P. Merkel, D.H. Miller, N. Neumeister, I. Shipsey, D. Silvers, A. Svyatkovskiy, M. Vidal Marono, H.D. Yoo, J. Zablocki, Y. Zheng

Purdue University Calumet, Hammond, U.S.A.

S. Guragain, N. Parashar

Rice University, Houston, U.S.A.

A. Adair, B. Akgun, C. Boulahouache, K.M. Ecklund, F.J.M. Geurts, W. Li, B.P. Padley, R. Redjimi, J. Roberts, J. Zabel

University of Rochester, Rochester, U.S.A.

B. Betchart, A. Bodek, Y.S. Chung, R. Covarelli, P. de Barbaro, R. Demina, Y. Eshaq, T. Ferbel, A. Garcia-Bellido, P. Goldenzweig, J. Han, A. Harel, D.C. Miner, D. Vishnevskiy, M. Zielinski

The Rockefeller University, New York, U.S.A.

A. Bhatti, R. Ciesielski, L. Demortier, K. Goulianos, G. Lungu, S. Malik, C. Mesropian

Rutgers, the State University of New Jersey, Piscataway, U.S.A.

S. Arora, A. Barker, J.P. Chou, C. Contreras-Campana, E. Contreras-Campana, D. Duggan, D. Ferencek, Y. Gershtein, R. Gray, E. Halkiadakis, D. Hidas, A. Lath, S. Panwalkar, M. Park, R. Patel, V. Rekovic, J. Robles, K. Rose, S. Salur, S. Schnetzer, C. Seitz, S. Somalwar, R. Stone, S. Thomas, M. Walker

University of Tennessee, Knoxville, U.S.A.

G. Cerizza, M. Hollingsworth, S. Spanier, Z.C. Yang, A. York

Texas A\&M University, College Station, U.S.A.

R. Eusebi, W. Flanagan, J. Gilmore, T. Kamon ${ }^{61}$, V. Khotilovich, R. Montalvo, I. Osipenkov, Y. Pakhotin, A. Perloff, J. Roe, A. Safonov, T. Sakuma, S. Sengupta, I. Suarez, A. Tatarinov, D. Toback 
Texas Tech University, Lubbock, U.S.A.

N. Akchurin, J. Damgov, C. Dragoiu, P.R. Dudero, C. Jeong, K. Kovitanggoon, S.W. Lee, T. Libeiro, I. Volobouev

Vanderbilt University, Nashville, U.S.A.

E. Appelt, A.G. Delannoy, C. Florez, S. Greene, A. Gurrola, W. Johns, P. Kurt, C. Maguire, A. Melo, M. Sharma, P. Sheldon, B. Snook, S. Tuo, J. Velkovska

University of Virginia, Charlottesville, U.S.A.

M.W. Arenton, M. Balazs, S. Boutle, B. Cox, B. Francis, J. Goodell, R. Hirosky, A. Ledovskoy, C. Lin, C. Neu, J. Wood

Wayne State University, Detroit, U.S.A.

S. Gollapinni, R. Harr, P.E. Karchin, C. Kottachchi Kankanamge Don, P. Lamichhane, A. Sakharov

University of Wisconsin, Madison, U.S.A.

M. Anderson, D. Belknap, L. Borrello, D. Carlsmith, M. Cepeda, S. Dasu, E. Friis, L. Gray, K.S. Grogg, M. Grothe, R. Hall-Wilton, M. Herndon, A. Hervé, P. Klabbers, J. Klukas, A. Lanaro, C. Lazaridis, R. Loveless, A. Mohapatra, M.U. Mozer, I. Ojalvo, F. Palmonari, G.A. Pierro, I. Ross, A. Savin, W.H. Smith, J. Swanson

\author{
$\dagger$ : Deceased \\ 1: Also at Vienna University of Technology, Vienna, Austria \\ 2: Also at CERN, European Organization for Nuclear Research, Geneva, Switzerland \\ 3: Also at National Institute of Chemical Physics and Biophysics, Tallinn, Estonia \\ 4: Also at Universidade Federal do ABC, Santo Andre, Brazil \\ 5: Also at California Institute of Technology, Pasadena, U.S.A. \\ 6: Also at Laboratoire Leprince-Ringuet, Ecole Polytechnique, IN2P3-CNRS, Palaiseau, France \\ 7: Also at Suez Canal University, Suez, Egypt \\ 8: Also at Zewail City of Science and Technology, Zewail, Egypt \\ 9: Also at Cairo University, Cairo, Egypt \\ 10: Also at Fayoum University, El-Fayoum, Egypt \\ 11: Also at British University in Egypt, Cairo, Egypt \\ 12: Now at Ain Shams University, Cairo, Egypt \\ 13: Also at National Centre for Nuclear Research, Swierk, Poland \\ 14: Also at Université de Haute-Alsace, Mulhouse, France \\ 15: Also at Joint Institute for Nuclear Research, Dubna, Russia \\ 16: Also at Moscow State University, Moscow, Russia \\ 17: Also at Brandenburg University of Technology, Cottbus, Germany \\ 18: Also at The University of Kansas, Lawrence, U.S.A. \\ 19: Also at Institute of Nuclear Research ATOMKI, Debrecen, Hungary \\ 20: Also at Eötvös Loránd University, Budapest, Hungary \\ 21: Also at Tata Institute of Fundamental Research - HECR, Mumbai, India \\ 22: Now at King Abdulaziz University, Jeddah, Saudi Arabia \\ 23: Also at University of Visva-Bharati, Santiniketan, India \\ 24: Also at Sharif University of Technology, Tehran, Iran
}


25: Also at Isfahan University of Technology, Isfahan, Iran

26: Also at Shiraz University, Shiraz, Iran

27: Also at Plasma Physics Research Center, Science and Research Branch, Islamic Azad University, Tehran, Iran

28: Also at Facoltà Ingegneria Università di Roma, Roma, Italy

29: Also at Università della Basilicata, Potenza, Italy

30: Also at Università degli Studi Guglielmo Marconi, Roma, Italy

31: Also at Università degli Studi di Siena, Siena, Italy

32: Also at University of Bucharest, Faculty of Physics, Bucuresti-Magurele, Romania

33: Also at Faculty of Physics of University of Belgrade, Belgrade, Serbia

34: Also at University of California, Los Angeles, Los Angeles, U.S.A.

35: Also at Scuola Normale e Sezione dell' INFN, Pisa, Italy

36: Also at INFN Sezione di Roma, Roma, Italy

37: Also at University of Athens, Athens, Greece

38: Also at Rutherford Appleton Laboratory, Didcot, United Kingdom

39: Also at Paul Scherrer Institut, Villigen, Switzerland

40: Also at Institute for Theoretical and Experimental Physics, Moscow, Russia

41: Also at Albert Einstein Center for Fundamental Physics, Bern, Switzerland

42: Also at Gaziosmanpasa University, Tokat, Turkey

43: Also at Adiyaman University, Adiyaman, Turkey

44: Also at Izmir Institute of Technology, Izmir, Turkey

45: Also at The University of Iowa, Iowa City, U.S.A.

46: Also at Mersin University, Mersin, Turkey

47: Also at Ozyegin University, Istanbul, Turkey

48: Also at Kafkas University, Kars, Turkey

49: Also at Suleyman Demirel University, Isparta, Turkey

50: Also at Ege University, Izmir, Turkey

51: Also at School of Physics and Astronomy, University of Southampton, Southampton, United Kingdom

52: Also at INFN Sezione di Perugia; Università di Perugia, Perugia, Italy

53: Also at Utah Valley University, Orem, U.S.A.

54: Now at University of Edinburgh, Scotland, Edinburgh, United Kingdom

55: Also at Institute for Nuclear Research, Moscow, Russia

56: Also at University of Belgrade, Faculty of Physics and Vinca Institute of Nuclear Sciences, Belgrade, Serbia

57: Also at Argonne National Laboratory, Argonne, U.S.A.

58: Also at Erzincan University, Erzincan, Turkey

59: Also at Mimar Sinan University, Istanbul, Istanbul, Turkey

60: Also at KFKI Research Institute for Particle and Nuclear Physics, Budapest, Hungary

61: Also at Kyungpook National University, Daegu, Korea 